\title{
Entre application des normes nationales et gestion optimale des ressources: le cas des farines animales dans le contexte de l'Encéphalopathie Spongiforme Bovine (ESB) en France (2000-2009)
}

\author{
Between application of national standards and optimal management of \\ resources: the case of feed-bone meal in the context of Bovine
} Spongiform Encephalopathy (BSE) in France (2000-2009)

\author{
Alexandre Geffroy ${ }^{1}$ \\ ${ }^{1}$ Ingénieur de recherche en Développement Durable et Responsabilité Sociétale, Université de Rouen Normandie
}

RÉSUMÉ. A partir de l'étude de la gestion des farines animales dans le contexte de l'Encéphalopathie Spongiforme Bovine (ESB), cet article propose de questionner l'importance des dimensions scalaires, territoriales et temporelles d'une crise par l'analyse des actions conduites par les Préfets de département. Ces derniers ont, en effet, été saisis par l'Etat central pour conduire les premières actions de gestion des farines animales à la suite de leur interdiction complète dans l'alimentation des animaux d'élevage. Une stratégie nationale de gestion avait, à ce titre, été préalablement éditée par les services de l'Etat central, l'action locale devant être théoriquement conforme à ce cahier des charges. Or, il s'avère qu'à la lecture des archives gouvernementales, certains préfets n'aient pas respecté à la lettre ces prescriptions. II s'agit, de la sorte, de mesurer quelles ont pu être les conséquences, notamment territoriales et temporelles, de ces adaptations locales au plan national et d'émettre des pistes explicatives de ces décisions. Dans cette optique, les modèles de localisation optimale peuvent s'avérer être de très bons outils pour évaluer l'importance et les conséquences des actions prises localement dans la gestion d'une crise nationale.

ABSTRACT. Based on the study of the French management of feed-bone meal in the context of Bovine Spongiform Encephalopathy (BSE), this article aims to question the importance of the scalar, territorial and temporal dimensions of a crisis by analysing the actions carried out by French departmental prefects. They were the ones were in charge to carry out the first actions for the management of feed-bone meal just after their total ban in the feeding of livestock. To manage this, a national management strategy was published by the central government. Local actions should theoretically comply with these specifications, but it appears from the government's archives that some prefects did not really respect these prescriptions. Therefore, the aim is to measure the consequences, particularly territorial and temporal, of these local adaptations at the national level, and to suggest possible explanations of these decisions. From this point of view, optimal location models can prove to be very good tools to evaluate the importance and consequences of local actions in the management of a national crisis.

MOTS-CLÉS. ESB, Farines animales, France, Gestion de crise, Localisation optimale.

KEYWORDS. BSE, Feed-bone meal, France, Crisis management, Optimal location.

\section{Introduction ${ }^{1}$}

Les crises, par les problèmes qu'elles soulèvent et les impératifs de gestion qu'elles imposent, nécessitent l'activation et la mise en relation de ressources identifiées utiles par les instances en charge de les administrer (Robert J., 2012 ; Metzger et al., 2013 ; Robert J. \& D’Ercole R., 2014).

\footnotetext{
${ }^{1}$ L'ensemble des données relatives aux volumes de farines animales produites, stockées et éliminées et aux processus et stratégies de gestion sont issues soit des Archives Nationales de Pierrefitte-sur-Seine (93), fonds « Mission Interministérielle pour l'Elimination des Farines Animales ", soit d'entretiens menés auprès de différents acteurs ayant participé à la gestion de cette crise (Geffroy, 2018).
} 
Ces ressources peuvent aussi bien être des acteurs que des lieux (Lajarge et al., 2012 ; Lacquement \& Chevalier P., 2016). La gestion de crise, entendue comme tel, se caractérise ainsi par l'élaboration de stratégies, l'édiction de normes, la mobilisation et la coordination d'acteurs situés à des niveaux d'action (infranational, national, supranational) potentiellement différents et des lieux potentiellement éloignés les uns des autres. Elle interroge, de ce fait, l'importance des échelles et des pratiques des acteurs «gestionnaires» qui, durant un certain laps de temps, agissent collectivement au sein d'une arène commune (Gilbert, 2002) afin de résorber une ou des perturbation(s). La mobilisation de ces ressources en situation de crise est, en outre, marquée par un contexte d'urgence et d'incertitude lié au côté brutal, disruptif de ces situations qui bouleversent l'ordre établi (Dautin et al, 2006). Les crises sont, en ce sens, des événements sociaux qui interrogent les capacités de réponses des sociétés concernées (Beck, 2006). Ainsi, en tant que phénomène endogène, l'évolution et la durée d'une crise sont déterminées par l'action des maillons humains mobilisés pour y faire face (Dubois-Maury \& Chaline, 2004). Il est, de la sorte, primordial de questionner le rôle des acteurs et des lieux de la gestion dans le traitement d'une crise (November, 2002), en particulier ceux mis en jeu dans les premiers instants puisque ce sont ces premières ressources qui influencent la poursuite des événements.

L'enjeu de cet article est alors de saisir l'importance et les conséquences, notamment temporelles, des stratégies et actions menées dans les premiers temps d'une crise : celles-ci sont-elles favorables à la résorption rapide des perturbations ou, au contraire, concourent-elles au maintien et/ou à l'aggravation des problèmes à administrer ? Pour mener à bien ce projet, l'article propose, d'une part, d'analyser la première phase de la gestion des farines animales (Geffroy \& Eliot, 2019) suite à leur interdiction complète dans l'alimentation des animaux d'élevage et, d'autre part, d'observer les conséquences de cette dernière dans les phases suivantes de la gestion. Cette décision forte, prise le 14 novembre 2000 pour lutter contre l'Encéphalopathie Spongiforme Bovine (ESB), plaça l'Etat français dans une situation de vulnérabilité puisque malgré l'interdiction, la production des farines animales demeura et demeure encore obligatoire car elles permettent de réduire en masse et en volume les déchets animaux non consommés par l'Homme. Ainsi, tant que de la viande sera produite, des farines le seront et ce sont, en moyenne, 8000 tonnes de ce produit carné qui sont produites chaque semaine en France. Le gouvernement dut donc, pratiquement du jour au lendemain, trouver des lieux en capacité d'entreposer de façon la plus sûre ces milliers de tonnes le temps que de nouveaux exutoires leurs soient trouvés. Des normes d'entreposages et des stratégies de localisation de ces sites furent nationalement adoptées tandis que le pouvoir de sélection fut octroyé aux préfets de département (Geffroy et Eliot, 2019). L'analyse des archives gouvernementales de l'époque (2000-2005) met en lumière que les sites localement sélectionnés par les différents préfets ne respectent pas tout ou partie des prescriptions nationales (Geffroy, 2018). L'article propose, dans ce contexte, de mesurer l'importance des décisions prises par les préfets dans les premiers temps de la gestion de la crise et leurs conséquences éventuelles dans l'évolution et la durée du traitement de ce problème sanitaire national. Pour cela, l'article confronte la localisation des sites réellement mobilisés par les préfets à un scénario de localisation optimale respectant scrupuleusement le cahier des charges établi par l'Etat central. A partir de ces comparaisons de localisation, l'article suggère, d'une part, des pistes explicatives pour comprendre pourquoi cette crise fut marquée par une gestion aussi longue et complexe et, d'autre part, fournit une piste de réflexion éventuelle pour prévenir et anticiper les problèmes de gouvernance qui eurent lieu. La gouvernance est ici entendue comme « un processus de coordination d'acteurs, de groupes sociaux et d'institutions, en vue d'atteindre des objectifs définis et discutés collectivement » (Le Galès, 2013). In fine, cette démarche d'analyse cherche à mieux comprendre des processus inhérents à la territorialisation de l'action publique (Sack, 1986 ; Peluso, 2005 ; Basset et Gautier, 2014 ; Gonin, 2016) en situation de crise, entendue comme une stratégie territoriale déployée par le gouvernement central pour contrôler une ou des ressource(s) nécessaire(s) à la résorption d'une perturbation. 


\section{Reconstruire la temporalité de la crise des farines animales à partir des archives gouvernementales : contexte, stratégie nationale envisagée, niveaux d'actions mobilisés}

L'ESB, maladie animale découverte pour la première fois en 1985, est reconnue comme un problème de santé publique depuis 1996, date à laquelle son lien avec la variante de la maladie de Creutzfeldt-Jakob (vMCJ) fut avéré. Dans ce contexte, les autorités françaises et européennes développèrent différentes mesures pour endiguer l'épizootie et réduire les risques d'exposition des consommateurs, le risque de transmission de la vMCJ étant lié au fait d'ingérer de la viande de bœuf contaminée par l'ESB. Au cœur de ces mesures se trouvaient les farines animales. En effet, ces produits carnés, utilisés dans l'alimentation des animaux d'élevage en tant que compléments protéinés, furent identifiés dès décembre 1987 comme responsables de la diffusion de l'agent pathogène, le prion, dans les troupeaux. En tant que problème communautaire, 1'ESB donna lieu à des procédures de gestion nationales différentes (Geffroy, 2018). Dans le cadre de cet article, seule la gestion française des farines animales est abordée. Pour cela, l'article se repose sur l'exploitation croisée d'archives textuelles, issues du fonds de la Mission Interministérielle pour l'Elimination des Farines Animales (MIEFA) ${ }^{2}$, entreposé aux sites des Archives nationales de Pierrefitte-sur-Seine, et d'archives orales, issues de 10 entretiens semi-directifs conduits auprès de divers acteurs, publics et privés, français et étrangers, ayant participé aux actions de résorption des perturbations.

\begin{tabular}{|c|c|c|c|}
\hline \multicolumn{2}{|l|}{ Archives écrites } & \multicolumn{2}{|c|}{ Archives orales } \\
\hline \multicolumn{2}{|l|}{$\begin{array}{c}\text { Archives MIEFA } \\
\text { (nombre de document(s) par type de document) }\end{array}$} & \multicolumn{2}{|c|}{ Entretiens avec des acteurs publics } \\
\hline $\begin{array}{c}\text { Rapports d'évaluation des actions entreprises ou à } \\
\text { entreprendre }\end{array}$ & 1 & \multicolumn{2}{|c|}{$\begin{array}{c}\text { Chargé de mission de l'Agence De l'Environnement et de la } \\
\text { Maîtrise de l'Energie (ADEME), spécialisé dans l'élimination des } \\
\text { déchets carnés, rattaché à la MIEFA }\end{array}$} \\
\hline $\begin{array}{l}\text { Lettres de contestation / d'information } \\
\text { d'associations / de citoyens }\end{array}$ & 5 & \multicolumn{2}{|c|}{$\begin{array}{l}\text { Cheffe de service FranceAgriMer, chargée de l'élimination des } \\
\text { farines animales après la fin d'activité de la MIEFA }\end{array}$} \\
\hline $\begin{array}{l}\text { Courrier entre le Préfet de la Zone de Défense } \\
\text { Ouest et la MIEFA }\end{array}$ & 1 & \multicolumn{2}{|c|}{$\begin{array}{l}\text { Entretiens avec des acteurs privés français } \\
\text { (nombre d'entretien(s) réalisé(s) par type d'opérateur) }\end{array}$} \\
\hline Brochettes informatives publiées par la MIEFA & 1 & Site de stockage & 4 \\
\hline $\begin{array}{l}\text { Réglementations, normes et textes de lois relatifs à } \\
\text { la production, le stockage et l'élimination }\end{array}$ & 3 & Cimenterie & 3 \\
\hline Statistiques relatives à la production, le stockage et & 20 & \multicolumn{2}{|c|}{ Entretien avec un acteur privé étranger } \\
\hline l'élimination des farines «à bas risque » & 32 & \multicolumn{2}{|c|}{ Directrice générale de Mindest } \\
\hline Total & 43 & Total & 10 \\
\hline
\end{tabular}

Figure 1. Corpus d'analyse ${ }^{3}$

\footnotetext{
${ }^{2}$ La MIEFA fut constituée suite à une lettre de mission du Premier ministre émise le 15 novembre 2000 auprès du préfet JeanPaul Proust, rapidement remplacé par le préfet Joël Lebeschu. Les missions confiées à la MIEFA furent, dans un premier temps, d'organiser une logistique d'urgence pour le stockage et l'élimination des farines animales « à bas risque » et, dans un second temps, de lancer des appels à proposition pour développer des infrastructures innovantes d'élimination thermique et alternative de ces dites farines. Aucun de ces projets n'a, cependant, abouti (Geffroy, 2018). La MIEFA était composée de 5 personnes : le préfet en chef chargé de la présidence de la MIEFA, une secrétaire, deux hauts fonctionnaires (un inspecteur général de l'Agriculture et un inspecteur général de l'Environnement) chargés des missions d'inspection, d'audit, de contrôle, de conseil et d'évaluation des actions à mener et des projets soumis et un agent de l'ADEME spécialisé dans l'élimination des produits carnés.

${ }^{3} 165$ documents furent, au total, extraits des archives nationales pour un volume de 564 pages. Or, un nombre conséquent d'entre eux s'avéra ne revêtir que peu d'importance pour analyser la localisation, les stratégies et la coordination de la gestion. 

prononcée (1989-2000)

Le premier cas d'ESB identifié en France fut découvert le 2 mars 1991 dans un élevage des Côtes-d'Armor. Pour autant, l'Etat n'attendit pas de découvrir ce premier cas pour mener des actions de gestion sur son territoire, en particulier à l'encontre des farines animales.

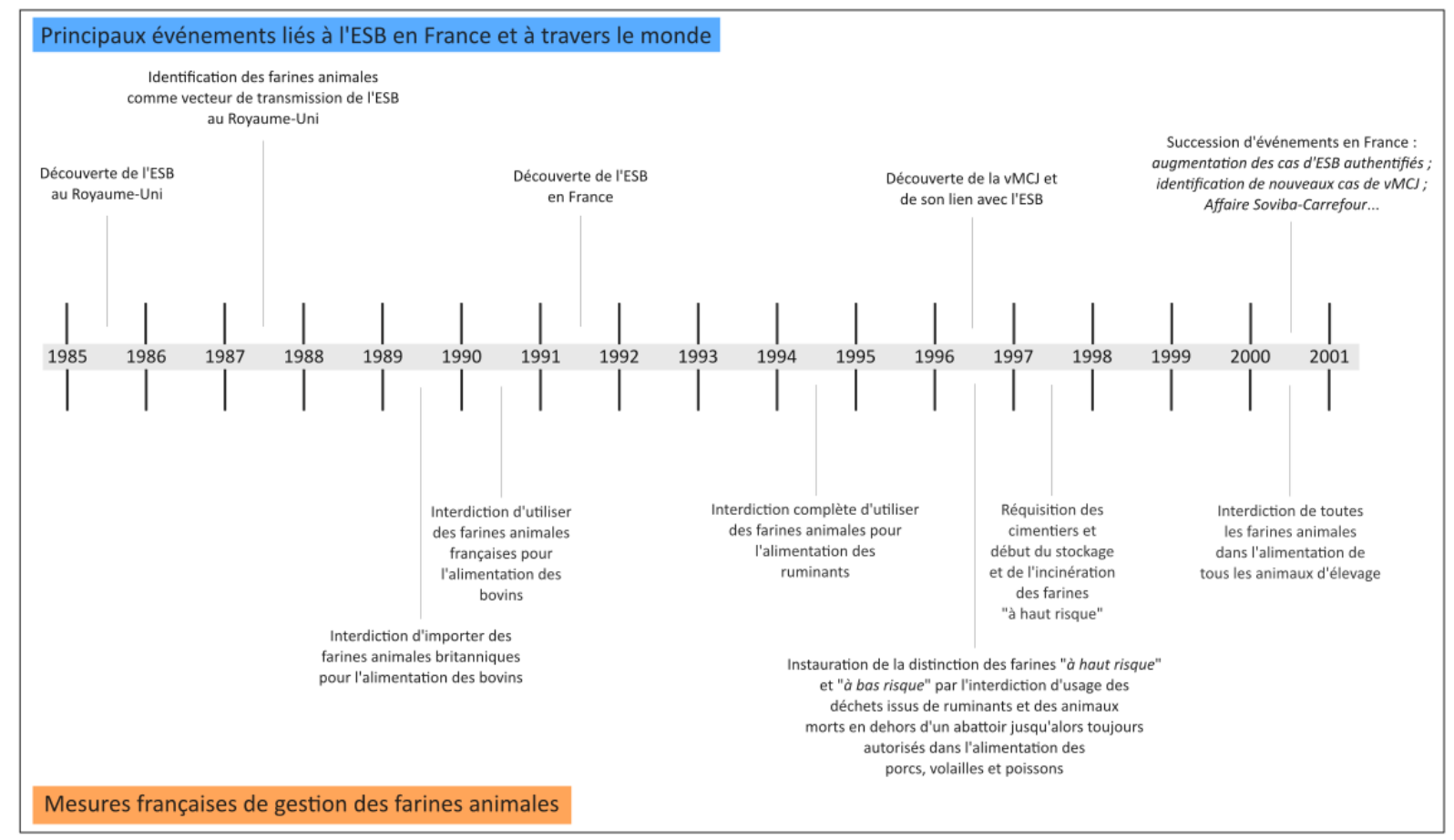

Figure 2. Chronologie des mesures de gestion prises en France à l'encontre des farines animales

Notant l'émergence d'un problème sanitaire de grande ampleur au Royaume-Uni, le gouvernement édicta le 13 août 1989 sa première mesure de gestion relative aux farines animales. Cet arrêté interdit l'importation des farines animales en provenance du Royaume-Uni dans l'alimentation exclusive des bovins. Cette mesure fut renforcée en juillet 1990 par l'interdiction d'utiliser désormais les farines animales produites en France dans l'alimentation, toujours exclusive, des bovins.

C'est en 1994, plus précisément le 20 décembre, que les farines animales furent définitivement retirées de l'alimentation de tous les ruminants (bovins, ovins et caprins), les seuls susceptibles, en l'état des connaissances scientifiques de l'époque, de contracter la maladie. Malgré ces mesures prohibitives, les déchets de ces ruminants étaient, à cette date, toujours utilisés, sous forme de farines, dans l'alimentation des porcs, volailles et poissons. Il fallut attendre l'annonce du premier ministre britannique, John Major, le 21 mars 1996 sur l'identification de la vMCJ et de son lien avec

C'est pourquoi seuls 43 documents ont été retenus dans le corpus d'analyse. Les archives textuelles permirent principalement de connaître les acteurs et lieux mobilisés ainsi que les normes infrastructurelles adoptées pour l'entreposage des farines animales. Les entretiens vinrent, en ce sens, compléter et prolonger les archives de la MIEFA par la compréhension des modalités d'organisation et de coordination des niveaux d'action et par l'explicitation des stratégies des acteurs. A cet égard, les critères qui ont sous-tendu la sélection des acteurs à enquêter relèvent de la localisation géographique et des volumes qu'ils furent amenés à gérer. Les questions que soulevaient ces critères étaient d'observer s'il y avait eu des pratiques de gestion différentes en fonction des contextes locaux. Enfin, du fait de la diversité des acteurs, de leur disponibilité et de leur localisation sur l'ensemble du territoire métropolitain, voire au-delà, le discours des enquêtés a été recueilli soit par entretien téléphonique, soit par entretien physique. 
l'ESB, pour que des décisions excluent les cadavres d'animaux morts en dehors d'un abattoir et les déchets de ruminants des cycles de production destinés à l'alimentation des porcs, volailles et poissons. Cette mesure instaura, de cette façon, une distinction entre farines dites «à haut risque » car issues d'animaux susceptibles d'être porteurs de l'agent pathogène et farines dites «à bas risque » car issues d'animaux pour lesquels l'ESB n'a jamais été détectée. Les farines «à haut risque » devaient, dès lors, être incinérées. Pour ce faire, l'Etat réquisitionna en 1997 l'industrie cimentière, la seule disposant d'installations compatibles pour l'élimination du prion potentiellement présent dans les farines «à haut risque ». Cette décision devait donc mettre fin à la diffusion de l'agent pathogène puisque tous les matériaux à risque spécifié étaient exclus de l'alimentation.

Pour autant, l'année 2000, à l'instar de l'année 1996, symbolisa un tournant dans la gestion de l'ESB. En effet, un nouveau système de dépistage, reposant sur des tests automatiques sur les bovins entrant à l'abattoir et non plus uniquement sur l'observation des symptômes cliniques dans les élevages, est lancé au cours du mois de juin. Ces tests, désormais appliqués à grande échelle, conduisirent à un pic de découverte d'un grand nombre de cas en très peu de temps. Ces découvertes accrues, non expliquées méthodologiquement par le gouvernement (Wolfer, 2004), entraînèrent une vague de contestation de l'opinion publique à l'encontre des politiques de gestion jusqu'alors menées, laissant présager que rien n'avait été fait pour véritablement endiguer l'épizootie. En parallèle, d'autres événements survinrent, rendant la période de plus en plus anxiogène : deux nouveaux cas de vMCJ furent authentifiés dans la population; un tribunal ne condamna pas un directeur d'entreprise de fabrication d'alimentation animale suspecté de frauder; des lots de viande furent retirés des étales commerciales après authentification d'un cas d'ESB dans le troupeau dont elle était issue ; la chaîne de télévision M6 diffusa, en novembre, un reportage proposant de suivre l'agonie de patients britanniques. Ces événements donnèrent, de ce fait, l'impression que l'ESB était omniprésente.

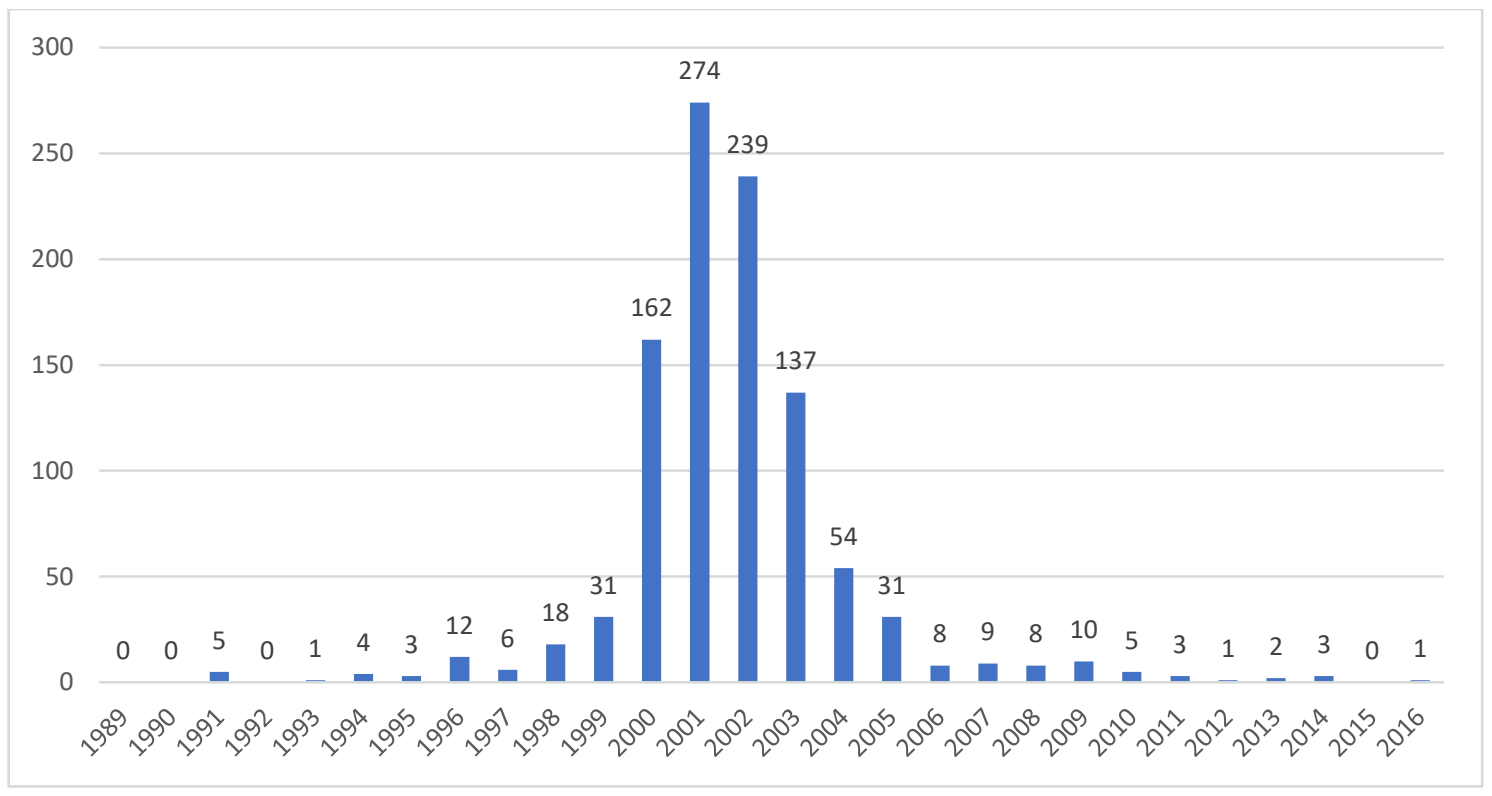

Figure 3. Nombre de cas d'ESB identifiés en France par année entre 1989 et $2016^{4}$

Malgré les tentatives de réassurance du gouvernement, une véritable psychose s'installa dans la population (Barbier, 2003). Les farines animales, encore autorisées dans l'alimentation des volailles

\footnotetext{
${ }^{4}$ Source : D'après les données de l'Organisation Mondiale de la Santé Animale (consultées en janvier 2021) :

https://www.oie.int/fr/sante-animale-dans-le-monde/situation-de-lesb-dans-le-monde-et-taux-dincidence-annuel/nombre-decas-dans-le-monde-hors-royaume-uni/.
} 
et des porcs, catalysèrent l'essentiel des craintes de l'opinion publique. La perception de la recrudescence des cas fut attribuée au maintien de ces produits dans les rations de ces animaux. Face à la contestation de l'action publique (Wolfer, 2004), la décision fut alors prise d'interdire toute valorisation alimentaire des farines animales le 14 novembre 2000. L'Union européenne suivit la décision de la France en étendant cette mesure à l'ensemble des Etats-membres le 4 décembre 2000. Le gouvernement dut donc, au lendemain de cette décision, composer avec une double difficulté de trouver à la fois des solutions de stockage et d'élimination des farines «à bas risque » tout en attendant que les volumes de farines «à haut risque », entreposés à la suite de la mesure prise en 1996, soient prioritairement détruits. En effet, la première année de réquisition des cimentiers fut consacrée à des campagnes de tests, ces derniers devant adapter leurs infrastructures, les farines étant un produit hautement volatile et pouvant encrasser leurs fours. L'élimination des farines ne débuta donc réellement qu'en 1998 et, en 2000, l'incinération conjointe des stocks résiduels de farines «à haut risque» et des farines fraichement produites ralentissait bien évidemment l'incinération des farines «à bas risque ». A ce titre, les farines «à haut risque » représentaient en $200232,5 \%$ des 800000 tonnes de farines produites en France, soit 260000 tonnes contre 540000 «à bas risque » $(67,5 \%)$.

\subsection{Une stratégie de gestion déconcentrée pour la recherche de solutions pour stocker les farines animales (2000-2002)}

La première étape de gestion de la crise dite des farines animales débuta, de la sorte, le jour suivant la déclaration de leur interdiction. L'enjeu premier fut d'administrer le problème posé par les 8000 tonnes de farines animales produites chaque semaine et qui n'étaient alors plus écoulées. Le Premier ministre, en collaboration avec les ministres de l'Agriculture et de l'Environnement, créa dans cette optique la Mission Interministérielle pour l'Elimination des Farines Animales (MIEFA) le 15 novembre 2000 et lui confia la mission d'organiser en temps réel le stockage et l'élimination de ces produits carnés.

Afin de désengorger les sites d'équarrissage, à savoir les producteurs de farines animales, la MIEFA devait disposer de lieux où entreposer ces produits le temps que de nouveaux exutoires leur soient trouvés. La MIEFA chargea, pour cela, les préfets de zone de défense et de sécurité de coordonner localement la sélection des sites de stockage. Cette première phase de gestion, conduite dans l'urgence et structurant la phase suivante d'élimination, fut menée dans une logique de déconcentration se manifestant par le transfert du pouvoir national vers des niveaux d'actions publics inférieurs (Rondinelli et al., 1983). Il fut, à ce titre, décidé de confier le stockage des farines animales uniquement à des Installations Classées pour la Protection de l'Environnement (ICPE), c'est-à-dire des entreprises ayant pour habitude de manipuler des matières susceptibles de générer des nuisances ou des dangers pour l'environnement. Pour trouver de tels sites, les préfets de zone de défense et de sécurité s'appuyèrent sur les préfets des départements de leur circonscription et sur les Directions Régionales de l'Industrie, de la Recherche et de l'Environnement (DRIRE) ${ }^{5}$, en charge de la délivrance du statut ICPE et possédant ainsi une connaissance très fine du terrain et des sites susceptibles de stocker les farines animales. Ils purent également être, dans des cas plus rares, conseillés par des établissements d'équarrissage ayant connaissance de sites disponibles. Ce fut notamment le cas dans le département du Rhône où un centre de stockage fut établi dans la banlieue lyonnaise grâce aux conseils du groupe SARIA. Dans la majorité des cas, ce sont, cependant, les préfets de département qui, sur conseil de la DRIRE, émirent parallèlement des appels d'offres publics et des réquisitions. Un centre de stockage, localisé dans la commune de Saint-Gérand dans le Morbihan, illustre ce deuxième procédé de mobilisation. Ce site fut réquisitionné par le préfet du

\footnotetext{
${ }^{5}$ Les DRIRE ont été réorganisées en 2009 et 2010. Leurs missions ont été, pour l'essentiel, reprises par les Directions Régionales de l'Environnement, de l'Aménagement et du Logement (DREAL).
} 
département à la suite d'une visite d'agents de la DRIRE qui constatèrent qu'un entrepôt neuf, parfaitement étanche, était apte à recevoir immédiatement des farines animales ${ }^{6}$. Quelle que soit la technique de mobilisation, une fois qu'un site était sélectionné par l'autorité préfectorale, sa mise en service était entérinée par des agents de la Direction Départementale des Services Vétérinaires (DDSV). Ces derniers contrôlaient la conformité de l'infrastructure par rapport aux normes sécuritaires émises par l'Institut National de l'Environnement Industriel et des Risques (INERIS) en septembre 1997 dans le contexte de l'interdiction des farines « à haut risque ( (Lödel, 1997). Les stocks de farines ne devaient, par exemple, pas dépasser sept à huit mètres de hauteur pour éviter le double risque d'échauffement et d'autocombustion et se situer à l'abri des entrées et circulations d'air pour éviter leur diffusion (Geffroy, 2018). Une fois un centre de stockage déterminé et validé, le préfet de département concerné remontait l'information au préfet de sa zone de défense et de sécurité qui, lui-même, informait en dernier lieu la MIEFA.

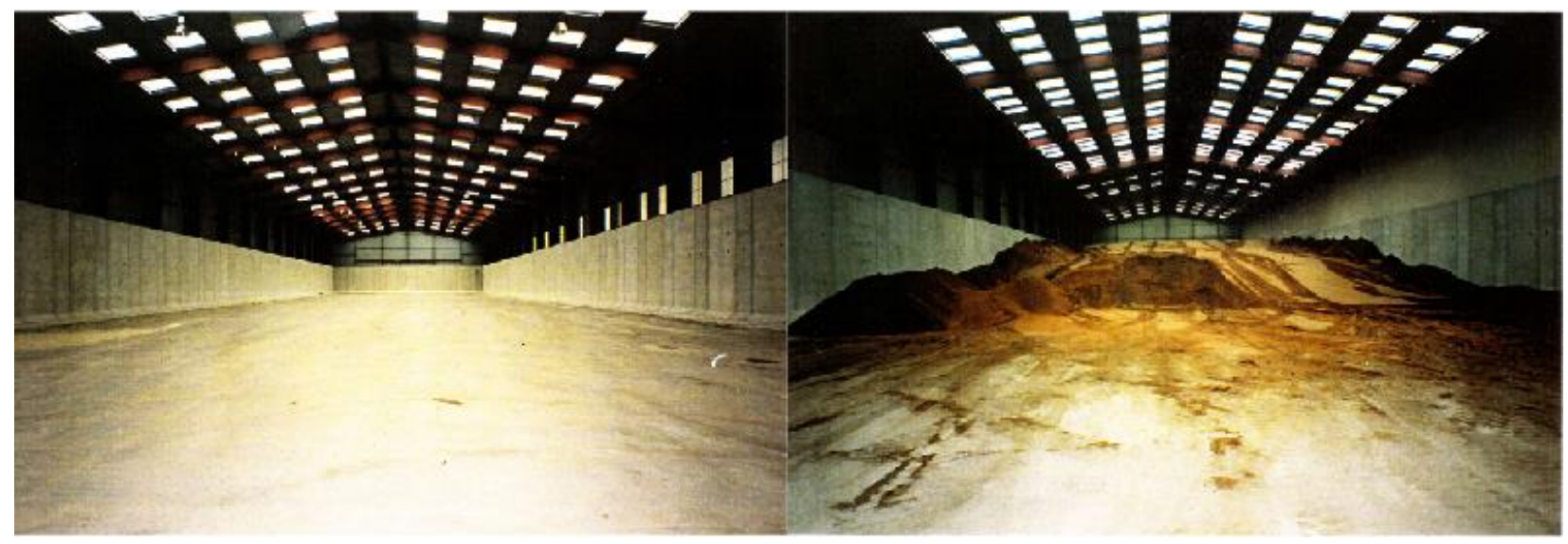

Photo 1. Exemple d'infrastructure mobilisée pour le stockage des farines dans la commune de Somsois dans la Marne ${ }^{7}$

A partir de ce moment, le site s'insérait dans un système de gestion nationale. Il devenait un intermédiaire temporaire dans le transit des farines entre leur lieu de production et leur lieu de stockage, les transferts de farine entre ces points étant coordonnés par la MIEFA. La localisation des sites était ainsi un véritable enjeu de gestion et, bien que le pouvoir de sélection fût octroyé à des acteurs locaux, ceux-ci devaient garder en mémoire la dimension nationale de la crise, d'une part, en percevant la dimension réticulaire de sa gestion et de sa logistique et, d'autre part, en respectant le cahier des charges établi par la MIEFA. Or, il s'avère que nombre de sites, en particulier les sites réquisitionnés, ne correspondent pas aux normes et à la stratégie envisagées par cette dernière.

\subsection{Des écarts dans l'application locale des directives nationales}

En effet, outre les normes sécuritaires propres à chaque site, la MIEFA souhaitait que chaque lieu sélectionné dispose d'une capacité minimale de stockage de 10000 tonnes de farines. Les sites de capacité inférieure étaient, au plan national, jugés inintéressants afin d'éviter la «prolifération » d'un trop grand nombre de stocks à travers le territoire métropolitain. La fixation du seuil de 10000 tonnes unitaires poursuivait donc un double objectif : réduire le nombre d'opérateurs engagés et,

\footnotetext{
${ }^{6}$ Informations tirées d'un entretien conduit le 28/06/2016 avec un employé du site de Saint-Gérand : « Notre entreprise a été réquisitionnée par la préfecture du département du Morbihan, par le biais des services de l'agriculture, en 2000, et parce que nous avions un entrepôt tout neuf et immédiatement disponible. »
}

${ }^{7}$ La dénomination des entreprises ayant participé au stockage des farines animales ne sera pas dévoilée. En effet, lors des entretiens, une entreprise a manifesté le souhait de voir les données qui lui sont rattachées être anonymisées. Nous avons donc décidé, par souci d'équité, d'anonymiser toutes les données relatives aux entreprises de stockage. 
grâce à cette minimisation, mieux encadrer et contrôler ces professionnels. Ce premier critère fut, à ce titre, peu respecté par les préfets de département puisque dix centres de stockage, sur les 26 mobilisés au total, avaient un tonnage disponible inférieur à ce seuil. La plupart des sites qui ne respectaient pas cette norme étaient des sites réquisitionnés durant les premiers mois qui suivirent l'interdiction de novembre 2000, révélant en cela l'urgence qui anima les préfets dans leur décision. Ainsi, sur les treize établissements réquisitionnés, sept avaient des capacités inférieures au minimum requis, la capacité moyenne des sites réquisitionnés étant de 11231 tonnes contre 59762 tonnes pour les entreprises mobilisés via les appels d'offres. 


\begin{tabular}{|c|c|c|c|c|}
\hline Commune & $\begin{array}{l}\text { Département } \\
\text { (ou pays le cas } \\
\text { échéant) }\end{array}$ & $\begin{array}{l}\text { Procédé de } \\
\text { sélection }\end{array}$ & $\begin{array}{l}\text { Capacité de } \\
\text { stockage } \\
\text { (en milliers de } \\
\text { tonnes) }\end{array}$ & $\begin{array}{c}\text { Volume entreposé } \\
\text { en octobre } 2003 \\
\text { (en milliers de } \\
\text { tonnes) }\end{array}$ \\
\hline Chateaubriant & Loire-Atlantique & Réquisition & 1000 & 1000 \\
\hline Jussy & Aisne & Réquisition & 2900 & 2900 \\
\hline Bouère & Mayenne & Appel d'offres & 5000 & 2500 \\
\hline $\begin{array}{l}\text { Saint-Saturnin-du- } \\
\text { Limet }\end{array}$ & Mayenne & Appel d'offres & 5000 & 5000 \\
\hline Guidel & Morbihan & Réquisition & 5000 & 5000 \\
\hline Pontivy & Morbihan & Réquisition & 5100 & 5100 \\
\hline Combrée & Maine-et-Loire & Réquisition & 6000 & 6000 \\
\hline Lizio & Morbihan & Appel d'offres & 6500 & 5700 \\
\hline $\begin{array}{l}\text { Sainte-Geneviève-des- } \\
\text { Bois }\end{array}$ & Loiret & Réquisition & 7000 & 7000 \\
\hline Montoire-de-Bretagne & Loire-Atlantique & Réquisition & 7500 & 7500 \\
\hline Nogent-sur-Vermisson & Loiret & Réquisition & 9000 & 3000 \\
\hline Saint-Gérand & Morbihan & Réquisition & 12000 & 12000 \\
\hline Caudan & Morbihan & Réquisition & 12000 & 600 \\
\hline Gand & France & Appel d'offres & 15000 & 3600 \\
\hline Pleine-Fougères & Île-et-Villaine & Réquisition & 20000 & 20000 \\
\hline Fougères & Île-et-Villaine & Appel d'offres & 20400 & 20400 \\
\hline Montoire-de-Bretagne & Loire-Atlantique & Réquisition & 22400 & 22400 \\
\hline Quincieux & Rhône & Réquisition & 27000 & 27000 \\
\hline Anvers & France & Appel d'offres & 30000 & 0 \\
\hline Coulombiers & Vienne & Appel d'offres & 40000 & 40000 \\
\hline Lyon & Rhône & Appel d'offres & 60000 & 16620 \\
\hline Somsois & Marne & Appel d'offres & 70000 & 52000 \\
\hline Plénée-Jugon & Côtes-d'Armor & Appel d'offres & 75000 & 75000 \\
\hline Châtillon-sur-Thouet & Deux-Sèvres & Appel d'offres & 110000 & 99000 \\
\hline Rogerville & Seine-Maritime & Appel d'offres & 150000 & 150000 \\
\hline Rogerville & Seine-Maritime & Appel d'offres & 190000 & 181100 \\
\hline
\end{tabular}

Figure 4. Capacité de stockage et volume stocké de farines animales «à bas risque » française en octobre 2003 (par ordre croissant des capacités de stockage) ${ }^{8}$

\footnotetext{
${ }^{8}$ Source : D'après des documents de suivi des stocks extraits des archives de la MIEFA. Pour consulter le tableau relatif au mois d'octobre 2003, voir l'annexe 31 de la thèse d'A. Geffroy (2018), La territorialisation de l'action publique en situation de crise. Le 
La MIEFA souhaitait, enfin, que la localisation des sites de stockage prenne en compte l'implantation géographique des équarrisseurs et des cimentiers de façon à réduire les distances de transit des farines animales. Ce critère visait à diminuer les risques d'accidents routiers et de diffusion des farines dans l'environnement, l'essentiel des flux s'effectuant par camions bennes fermés et bâchés. Une nouvelle fois, l'application de cette stratégie nationale au plan local a donné lieu à des décalages importants. Ce critère de minimisation des distances et d'intermédiarité spatiale des centres de stockage entre les lieux de production et d'élimination reposait sur une difficulté dans la mesure où la localisation de ces deux derniers acteurs ne coïncident que très peu. L'essentiel des usines d'équarrissage étaient et sont toujours implantées dans les principaux bassins d'élevage du Grand-Ouest (Bretagne, Pays-de-la-Loire) tandis que les cimenteries sont principalement localisées dans l'Est de la France. Il s'avère, de la sorte, que ce sont principalement les préfets de département de la zone de défense ouest qui participèrent à la sélection des sites de stockage. Cela conduisit à une concentration géographique des sites dans l'Ouest de la France, avec, par exemple, quatorze centres mobilisés uniquement en Bretagne et dans les Pays-de-la-Loire sur les vingt-six centres nationalement mobilisés, soit $54 \%$ du total. Deux sites furent, par ailleurs, sélectionnés en Belgique, témoignant eux aussi d'un décalage entre les stratégies nationales et les actions locales qui eurent cours.

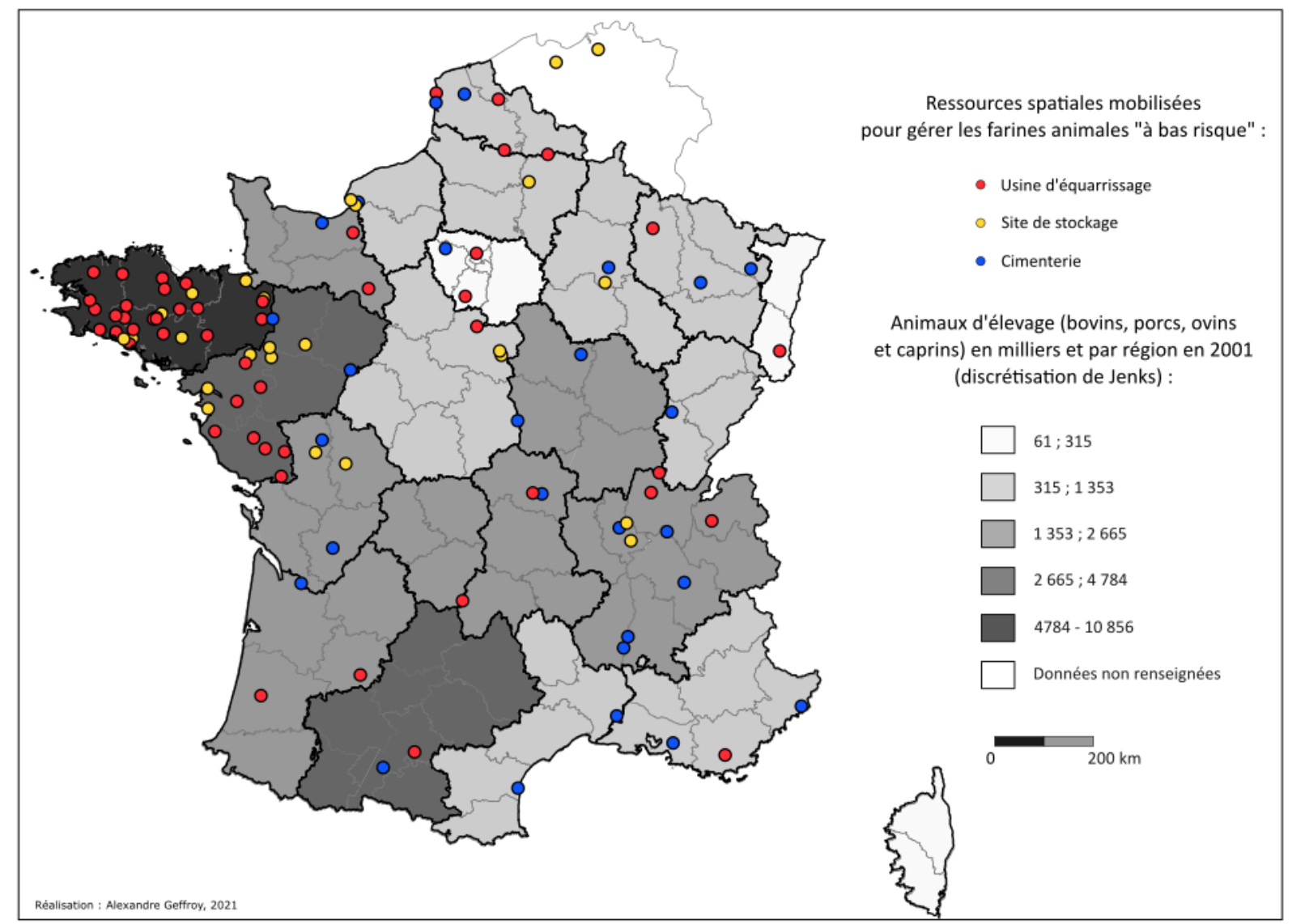

Figure 5. Localisation des 26 sites de stockage réels en activité à la fin de l'année $2003^{9}$

${ }^{9}$ Source : D'après les données d'Eurostat compilées dans le dossier « Structures des élevages » (consulté en mars 2021) : https://ec.europa.eu/eurostat/fr/web/agriculture/data/database. 


\subsection{Des premiers sites saturés entraînant des difficultés pour les phases suivantes de la gestion de la crise (2002-2009)}

Cette hyper-concentration dans l'ouest de la France des sites de stockage conduisit à des difficultés lors des phases suivantes de la gestion, notamment lorsqu'il fallut procéder à leur élimination à partir de 2003, année où les derniers stocks de farines «à haut risque » furent résorbés par les cimentiers. Or, c'est également au cours de l'année 2003 que l'Etat français cessa, d'une part, de réquisitionner les cimentiers et, d'autre part, de subventionner l'élimination des farines animales. Cette décision, motivée par l'amortissement des aménagements réalisés par les cimentiers depuis 1996 pour brûler les farines, entraîna le retrait de certains d'entre eux de ce nouveau marché. Les cimentiers ont, en effet, besoin, pour la production de ciments, de maintenir la flamme de leur four à une température de $2000^{\circ} \mathrm{C}$. Ils sont donc de grands consommateurs de déchets à fort pouvoir calorifique tels que des cokes de pétrole ou des pneumatiques usagés. Les farines animales, pour leur part, disposent d'un pouvoir calorifique bien moindre que les déchets pré-cités, leur incinération étant jugée intéressante par les cimentiers uniquement au regard de leur coût d'achat qui, de 1997 à 2003, était entièrement subventionné par l'Etat français. L'élimination en flux tendu des farines fraichement produites fut, à partir de cette date, confiée aux équarisseurs, les farines redevenant, malgré leur caractère potentiellement dangereux, une ressource marchande. Le prix des farines se mit alors à fluctuer selon les entreprises et les cimentiers durent, au-delà du coût d'achat des farines animales, prendre en compte les frais de transports pour acheminer celles-ci jusqu'à leurs installations. Cette problématique qui se posa dès 2003 pour l'élimination en flux tendu rentra également en ligne de compte pour l'élimination des stocks «à bas risque » mobilisés depuis novembre 2000. Le tonnage des farines animales incinérées par l'ensemble des cimenteries françaises baissa ainsi progressivement à partir de l'année 2003, année record pendant laquelle 382000 tonnes furent brûlées par ces opérateurs.

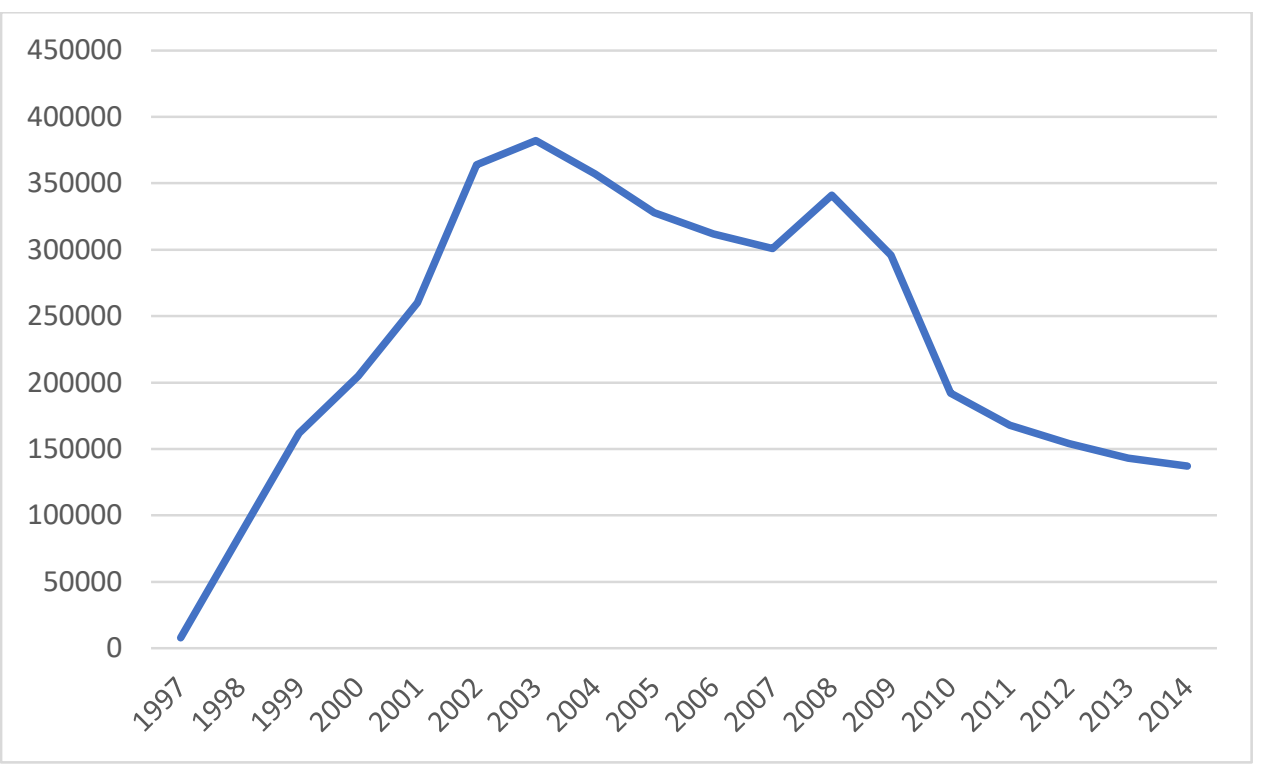

Figure 6. Volumes, en tonnes, de farines animales incinérées par les cimenteries françaises entre 1997 et 2014 (d'après l'Association Technique de l'Industrie des Liants Hydrauliques, 2015)

Les capacités d'élimination en flux tendu mirent, de ce fait, du temps à égaler les quantités produites par semaine. Les stocks de farines en attente de destruction continuèrent ainsi à progressivement augmenter, ceux-ci atteignant leur maximum en octobre 2003 avec 770420 tonnes simultanément entreposées (Geffroy et Eliot, 2019). C'est pourquoi la MIEFA entra en contact dès 2001 avec des opérateurs étrangers, notamment belges, allemands et néerlandais, pour amplifier les volumes éliminés en flux tendus. La mobilisation de deux sites de stockage en Belgique dut, en ce sens, être motivée par l'appétence de cimentiers belges pour les farines animales françaises. 
Le déstockage des farines «à bas risque » entreposées ne commença de façon infime qu'à partir de 2005. Ces premiers déstockages furent menés à l'échelle locale via les préfets des départements abritant un ou plusieurs centre(s) d'entreposage. Ces derniers étaient effectivement chargés d'établir des contrats avec des industriels souhaitant éliminer tout ou partie des volumes entreposés dans leur circonscription. Le déstockage complet de la vingtaine de sites en activité débuta, pour sa part, en 2006, année à partir de laquelle la gestion des farines animales fraichement produites ne posait plus de problèmes de gestion, et fut conduit à une échelle plus large. Ce fut, en effet, l'Office National Interprofessionnel de 1'Elevage et de ses Produits (ONIEP) ${ }^{10}$, venu en remplacement de la MIEFA ${ }^{11}$ dissoute peu de temps avant, qui se vit confier la tâche de mener les opérations de déstockage. Dans cette optique, l'ONIEP publia un appel d'offre national et européen pour vendre l'ensemble des farines stockées. C'est ainsi que le courtier suisse Mindest remporta «65\% des appels d'offre français $»^{12}$, les $35 \%$ restants étant achetés par plusieurs cimenteries françaises. La gestion de ces farines devint, à partir de la signature du contrat, la charge exclusive de cette société, les services déconcentrés de l'Etat n'intervenant plus que pour des opérations de surveillance et de contrôle des stocks, le temps que ceux-ci se résorbent. Mindest valorisa les farines animales françaises auprès de cimenteries françaises mais également auprès de centrales thermiques ou d'incinérateurs d'ordures ménagères allemands et italiens (Geffroy et Eliot, 2019). La fin de la gestion des farines animales fut ainsi marquée par un désengagement extrêmement prononcé des pouvoirs publics et un renoncement aux stratégies et normes de précaution élaborées lors de la première phase, les farines animales parcourant, pour certaines, des milliers de kilomètres entre leur lieu de stockage et leur lieu d'élimination. A ce titre, le travail se heurte ici à une difficulté d'accès à des informations exhaustives puisque des données relatives au transfert de farines de leur lieu de stockage à leur lieu d'élimination n'ont été trouvées que pour cinq sites : Plénée-Jugon dans les Côtes-d'Armor, SaintSaturnin-du-Limet dans la Mayenne, Rogerville en Seine-Maritime, Lyon dans le Rhône et Châtillon-sur-Thouet dans les Deux-Sèvres. Ces cinq sites représentaient $49 \%$ du total de farines nationalement entreposées en octobre 2003, soit 376720 tonnes. La distance moyenne séparant ces sites des cimenteries ayant réellement procédé à l'incinération des produits qu'ils entreposèrent est de 828 kilomètres, avec une distance maximale de 1268 kilomètres pour les farines stockées à SaintSaturnin-du-Limet. Mindest mit, par ailleurs, près de trois ans pour éliminer tous les sites, la destruction du dernier stockage en activité, situé à Rogerville, ayant eu lieu lors de l'année 2009.

\footnotetext{
${ }^{10}$ L'ONIEP n'existe plus depuis 2009, ses missions étant désormais portées par FranceAgriMer.

${ }^{11}$ Nous ne pouvons cependant pas apporter de précisions supplémentaires quant à la date exacte de dissolution de la MIEFA. Aucune information n'a été trouvée à ce sujet, que ce soit aux Archives nationales ou au cours de notre entretien mené avec un ancien membre de l'ADEME détaché auprès de la MIEFA entre janvier 2001 et juin 2002.

${ }^{12}$ Citations in « Mindest - Société », site internet de l'entreprise : http://www.mindest.ch/societe-mindest.php?LANG=FRA 


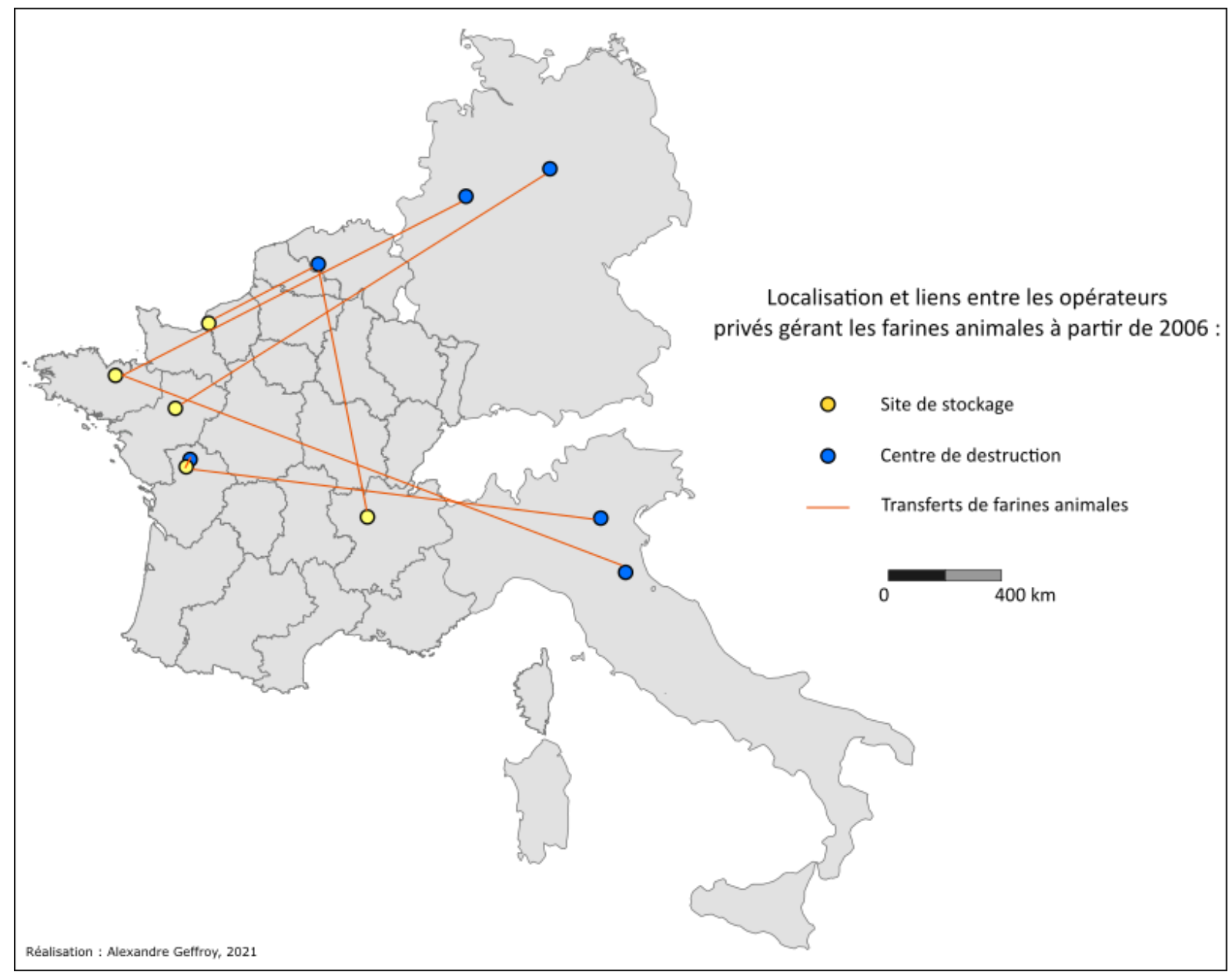

Figure 7. Illustration de transferts réels de farines animales «à bas risque » de leur lieu de stockage à leur lieu d'élimination

Il s'avère donc que le principe de localisation qui fut majoritairement appliqué par les services déconcentrés de l'Etat soit celui de la proximité géographique des centres de stockage aux usines d'équarrissage. Ces pratiques, ne prenant que partiellement en compte la stratégie de gestion élaborée par l'Etat central, semblent être porteuses des germes qui conduisirent à une si longue et si complexe élimination des stocks de farines. L'aspect territorial de la gestion de crise, et sa dimension nationale, semblent avoir été insuffisamment perçus. L'attribution du pouvoir de sélection à des acteurs locaux, en l'occurrence les préfets de départements, laisse présager qu'aucun mécanisme de coordination ou de solidarité nationale n'ait émergé entre territoires en prise directe avec le problème de la production des farines animales et territoires sans usines d'équarrissage dans leur circonscription puisque les sites de stockage furent, pour l'essentiel, mobilisés dans le Grand Ouest. Il ne semble ainsi pas y avoir eu de concertation nationale, les sites de stockage n'ayant pas été, à cette première phase de gestion, perçus comme un ensemble interdépendant, comme des territoires multisitués (Cortes et Pesche, 2013) s'insérant dans une problématique nationale commune. Pour confronter cette hypothèse qui sous-tend que les adaptations locales à la stratégie nationale aient entraîné des perturbations pour la résorption de la crise, il conviendrait d'observer quelle aurait pu être la localisation optimale, du moins théoriquement, des sites de stockage si les normes avaient toutes été respectées à la lettre, si la gestion de ce problème n'avait pas été menée dans l'urgence. A ce titre, les résultats de la modélisation permettront, ou non, de conforter notre hypothèse qui, en l'état, ne s'appuie que sur des informations parcellaires au regard de l'incomplétude des archives accessibles et de la difficulté à mener des entretiens avec les acteurs impliqués (Geffroy, 2018). 


\section{Les modèles de localisation-affectation : des outils utiles pour mesurer a posteriori les décalages induits par les adaptations locales aux normes nationales}

Les modèles de localisation-affectation peuvent, dans ce cadre, être des outils extrêmement intéressants aussi bien dans l'anticipation d'une crise que dans une lecture critique a posteriori puisqu'ils cherchent, via des algorithmes mathématiques, à optimiser la localisation de ressources (points d'offre) par rapport à un ensemble de lieux ou de personnes susceptibles d'utiliser celles-ci (points de demande). Dans le présent cas d'étude, les ressources sont les sites de stockage de farines animales tandis que les lieux susceptibles de les utiliser sont les usines d'équarrissage et les cimenteries. Cette famille de modèles propose, de la sorte, des outils qui s'avèrent utiles aussi bien dans une perspective de recherche, à l'instar du travail ici exposé, que dans une perspective d'aide à la décision ou d'aménagement $\mathrm{du}$ territoire. Ces modèles sont notamment utilisés pour la localisation des moyens de secours à personne (Souliès, 2015), d'unités de soins (Querriau et al., 2004), de maternités (De Ruffray et Hamez, 2009) ou encore de piscines publiques et de bureaux de poste (Beguin et $a l ., 1982$ ). Ils abordent, en ce sens, trois questions : «quels sont les besoins à satisfaire ? quelles sont les possibilités d'y répondre ? comment ajuster au mieux l'offre à la demande ? » (Beguin, 1995).



Figure 8. Éléments constitutifs d'un modèle de localisation affectation non pondéré

Dans le cadre de ce travail, le modèle spécifiquement utilisé est un modèle p-médian, calibré de façon à ce que tous ses paramètres répondent aux stratégies de l'Etat central. Les résultats fournis permettent ainsi d'observer si les adaptations effectuées par les préfets de département ont conduit à une localisation de la gestion bien différente de celle qu'elle aurait dû ou pu avoir si le cahier des charges national avait été respecté à la lettre. Il s'agit donc d'une analyse a posteriori mais ces modèles peuvent également se révéler très intéressants à l'amont d'une crise, dans une phase d'anticipation. Il aurait, par exemple, été possible d'avoir recours à ce type de modèle pour identifier les lieux sur le territoire métropolitain qui auraient pu permettre de réduire au maximum les distances de transit des farines «à bas risque » de leur lieu de production à leur lieu de stockage puis d'élimination, sachant que les normes utilisées au lendemain du 14 novembre 2000 étaient identiques à celles élaborées en 1997 pour gérer les farines «à haut risque ». Tous les critères, en dehors des volumes de farines qui allaient devoir être entreposés, étaient connus des autorités au moment de la proclamation de l'interdiction permettant, théoriquement, de cibler à l'amont les lieux idoines pour implanter les sites de stockage. 


\subsection{Le modèle p-médian : un modèle de localisation qui correspond aux volontés affichées par l'Etat central}

Plusieurs modèles de localisation-affectation existent: p-centre, modèles de couverture ou pmédian. Ces modèles répondent tous à une même suite d'opérations, leur différence provenant de leurs algorithmes d'optimisation qui ne poursuivent pas précisément les mêmes objectifs. Un modèle p-centre, par exemple, tend à localiser un point de manière à ce que la distance séparant ce point à tous les lieux nécessitant sa création soit la plus faible (Suzuki et Drezner, 1996). Un modèle de couverture, quant à lui, implique le calcul d'une zone autour de chaque lieu où un point doit être implanté (Baray, 2002). Cette zone, dite de couverture, tracée autour d'un lieu nécessitant la localisation d'une activité définit la distance maximale pour localiser un point. Enfin, le modèle pmédian minimise la somme pondérée des distances entre tous les points à localiser par rapport à ceux nécessitant sa création tout en s'assurant que chaque point créé soit affecté au point de demande le plus proche (Hakimi, 1964 ; Daskin, 1995 ; Peeters et Thomas, 1997). L'objectif de l'algorithme du p-médian semble, de la sorte, correspondre à la stratégie de proximité générale affichée par les autorités publiques nationales au début de la crise. Le choix d'un modèle dépend, en cela, du contexte auquel nous sommes confrontés (Querriau et al., 2004).

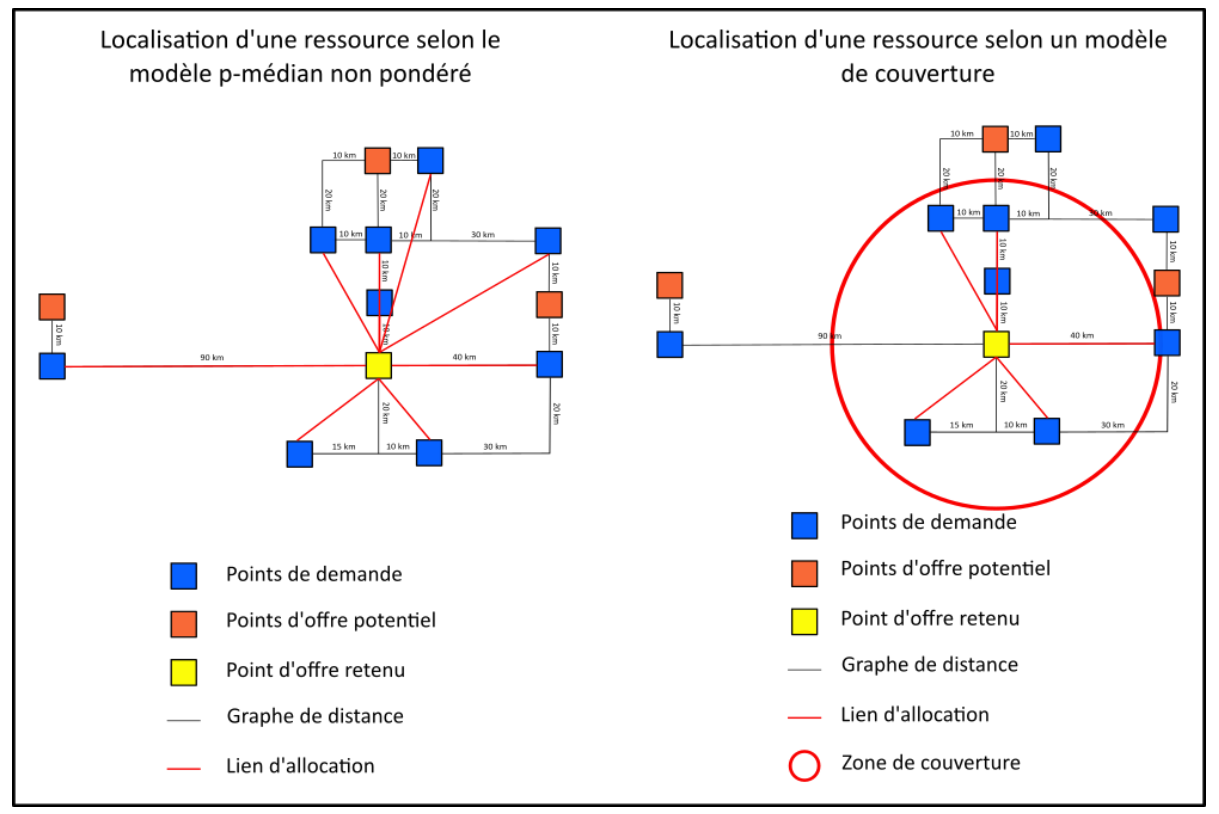

Figure 9. Illustration des principes de localisation d'une ressource selon deux modèles de localisation optimale

Le second attrait du p-médian pour le cas présent est que c'est un modèle de localisationaffectation discret, ce qui signifie que le nombre de ressources à localiser est déterminé par l'utilisateur et que la localisation des ressources varie selon le nombre de points à implanter. Chaque point a une incidence sur la sélection des sites restants car chaque sélection modifie la somme des distances entre points d'offre potentiels et points de demande. Par exemple, entre un scénario où dix sites sont à localiser et un second, utilisant les mêmes paramètres en entrée mais avec vingt sites à identifier, il n'est pas assuré que la totalité des dix sites du premier scénario soit sélectionné dans le second. Les modèles discrets sont ainsi plus réalistes (Beguin, 1995) que les modèles de localisation-affectation continus où le nombre de points à attribuer peut être infini. Le modèle utilisé a, de la sorte, été calibré de façon itérative afin d'identifier, calculs après calculs, le plus petit nombre de sites permettant d'accueillir le volume maximal de farines entreposées dans la réalité, à savoir 770420 tonnes.

Le modèle se heurte, toutefois, à une limite principale, à savoir la non-pondération du niveau de demande, c'est-à-dire les volumes de production de farines animales de chaque site d'équarrissage 
et les capacités d'élimination de chaque cimenterie. Cette limite est liée, une nouvelle fois, à l'incomplétude des données collectées et collectables. Les valeurs de distance ne sont, en ce sens, pas multipliées par les valeurs de poids assignées à chaque point de demande, tous les points de demande ayant le même poids dans notre modèle.

D’un point de vue méthodologique, la construction du modèle nécessite plusieurs étapes.

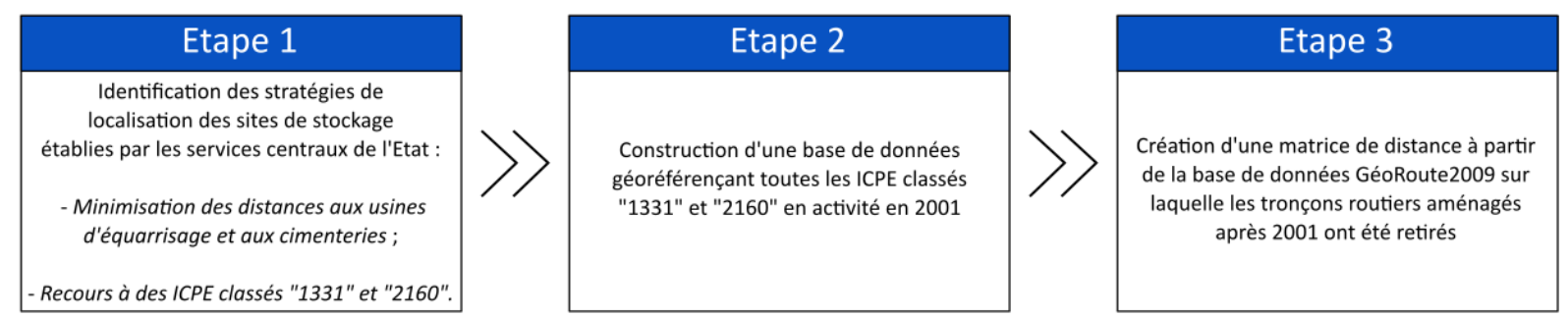

Figure 10. Les étapes de calibrage du modèle p-médian pour le calcul de la localisation optimale des sites de stockage des farines animales «à bas risque »

La première d'entre elles est d'intégrer, en plus de la localisation des usines d'équarrissage et des cimenteries, la localisation de toutes les infrastructures industrielles répondant aux critères établis par l'Etat. Pour cela, les archives de la MIEFA sont précieuses puisqu'elles permettent d'identifier que les 24 sites de stockage mobilisés sur le territoire français relèvent uniquement de deux nomenclatures $\mathrm{ICPE}^{13}$ pour lesquelles, en 2016, 2252 entreprises étaient référencées ${ }^{14}$. Ces données ne reflètent néanmoins pas les données du début des années 2000. Il faut, dans cette optique, garder dans l'analyse uniquement les installations ayant acquis le statut ICPE au plus tard en 2001. Prendre l'année 2001 plutôt que l'année 2000 se justifie par le fait que l'essentiel des sites de stockage mobilisés par les préfets le furent lors de cette année. Après cette procédure, 581 centres de stockages potentiels furent sélectionnés. Pour que le résultat obtenu par le modèle soit le plus proche des normes de l'Etat, il convient, ensuite, d'éliminer les sites ayant une capacité de stockage unitaire inférieure à 10000 tonnes de farines animales. Face à l'absence de données sur ce point précis, l'estimation de la capacité théorique d'entreposage s'appuie sur des données collectées lors de la visite de l'ancien site de stockage de Plénée-Jugon, dans les Côtes-d'Armor. Ce site eut, dans les faits, une capacité de stockage de 75000 tonnes de farines animales alors que le silo accueille, en temps normal, jusqu'à 100000 tonnes de blé. Le quotient obtenu de cette division est de 1,33. La base de données sur les ICPE renseigne, pour sa part, les capacités de stockage en mètre cube. Dans ce registre, le volume d'accueil de Plénée-Jugon indique une capacité maximale de $150000 \mathrm{~m}^{3}$. Le quotient entre $150000 \mathrm{~m}^{3}$ et 100000 tonnes de blé est cette fois égal à 1,5 . Si la pertinence de ces quotients peut être questionnée car ne s'appuyant que sur un seul exemple, ceux-ci sont, néanmoins, utilisés dans le calcul de toutes les capacités théoriques d'accueil. En effet, le travail se heurte à un accès extrêmement compliqué au terrain (Geffroy, 2018) et s'appuie donc sur des informations parcellaires. Quoi qu'il en soit, la marge d'erreur dans le cas présent a été jugée recevable car l'estimation s'appuie sur un relevé de terrain. Le quotient obtenu est, dès lors, probablement proche de la situation de tous les sites. A la suite de ces divisions, 379 centres potentiels de stockage furent implémentés dans le modèle dont l'essentiel est situé dans le centre et le Nord de la France et non pas en Bretagne ou dans les Pays-de-la-Loire, les deux régions pourtant les plus concernées dans les faits par la mobilisation de sites de stockage.

\footnotetext{
${ }^{13}$ Nomenclature 1331 relative aux entreprises employant ou stockant des produits toxiques comme des engrais et nomenclature 2160 relative aux silos de stockage de céréales, de grains et dégageant des poussières inflammables.

${ }^{14}$ https://www.georisques.gouv.fr/risques/installations/donnees\#/
} 




Figure 11. Localisation des 379 sites de stockage potentiels répondant aux critères de la stratégie nationale de gestion des farines animales

Il faut, par la suite, intégrer une matrice de distance pour que le modèle de localisation-affectation puisse déterminer l'éloignement entre tous les centres potentiels aux usines d'équarrissage et aux cimenteries. Cette matrice est ici construite à partir de la base de données Georoute 2009 de l'IGN ${ }^{15}$, laquelle fut préalablement amputée des segments routiers construits après 2001, afin de développer un scénario le plus en accord avec le contexte dans lequel s'est opérée l'action publique.

\subsection{Une localisation optimale théorique qui répartit mieux les sites de stockage sur l'ensemble du territoire métropolitain}

A partir de toutes ces données, il est possible de paramétrer le nombre de sites de stockage à sélectionner selon un procédé itératif. Le premier calcul visait, par exemple, à ne sélectionner que cinq sites. Ce premier scénario ne permit, cependant, pas d'identifier cinq sites en capacité d'entreposer 770420 tonnes de farines animales. Il s'avère que le minimum requis est estimé, par le modèle, à seize sites, soit dix de moins que dans la réalité. Les seize sites identifiés par le modèle auraient permis d'entreposer théoriquement 817273 tonnes de farines animales.

\footnotetext{
${ }^{15}$ Nous aurions, dans l'idéal, préféré conduire le travail sur la base de données Georoute 2001 mais les coûts nécessaires à son extraction par l'IGN étaient trop élevés. Nous nous sommes donc appuyés sur la base de données disponible au laboratoire et avons modifié uniquement les tracés autoroutiers, les réseaux de nationales et départementales n'ayant que très peu changé entre 2001 et 2009.
} 


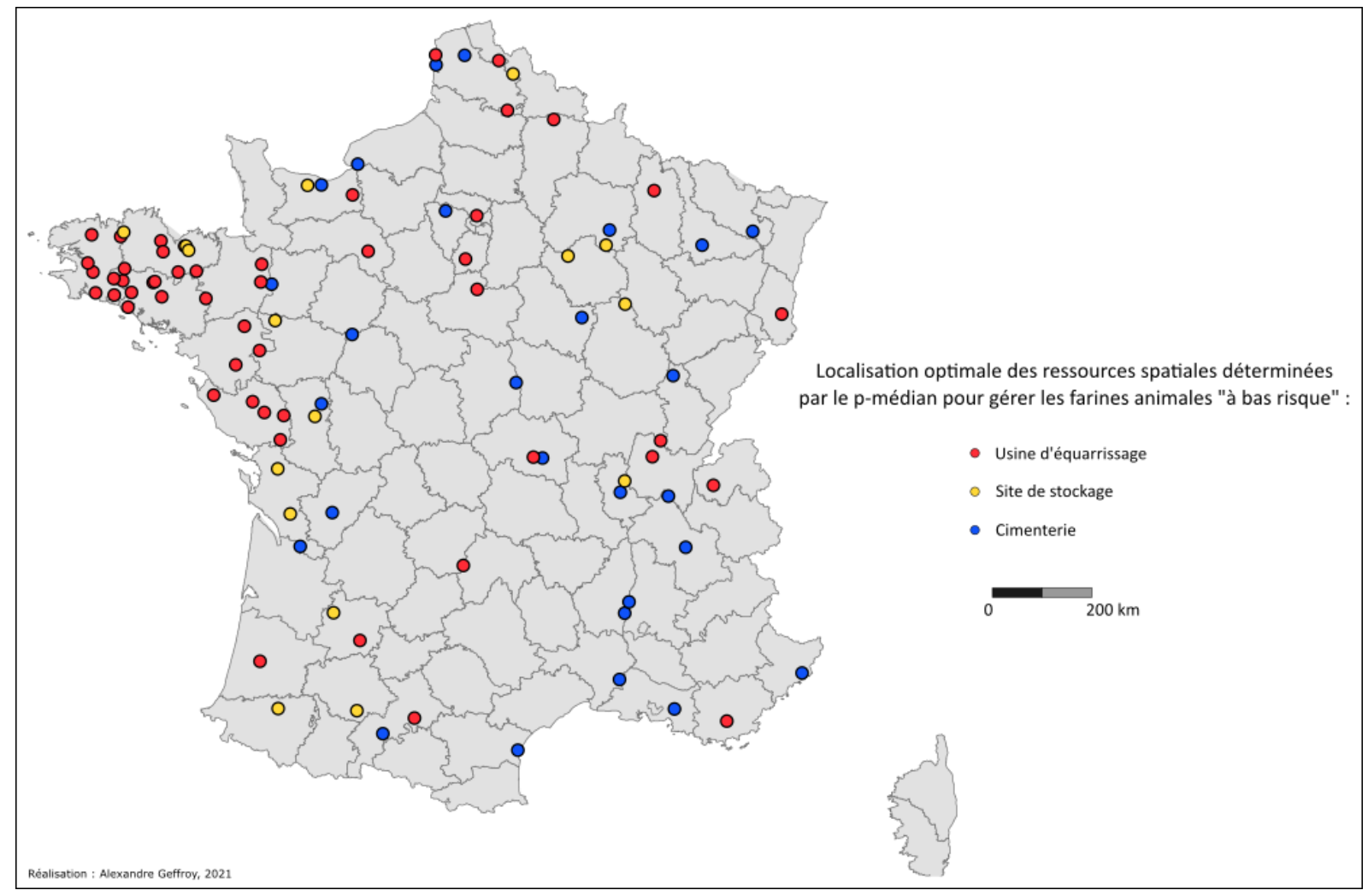

Figure 12. Localisation des seize sites de stockage " optimaux » permettant de répondre aux stratégies et enjeux de la gestion nationale des farines animales

La localisation optimale, à l'instar de la localisation réelle, tend à répartir la plupart des sites de stockage dans l'ouest de la France, plus précisément des ex-régions Basse-Normandie à Aquitaine. Il est également possible d'isoler un regroupement de trois sites dans l'est de la France, un dans le département de la Marne, un dans l'Aube et un en Côte-d'Or. Le modèle tend, de ce fait, à rapprocher le stockage des bassins de production, les distances moyennes séparant les sites de stockage identifiés par le modèle aux équarrisseurs $(384 \mathrm{~km})$ et aux cimenteries $(434 \mathrm{~km})$ étant relativement analogues à celles des sites réellement utilisés $(333 \mathrm{~km}$ et $442 \mathrm{~km})$. Le modèle est ainsi, sur certains aspects, proche de la réalité puisque les zones d'implantation sont pratiquement identiques sauf pour le sud-ouest et la Belgique. Les communes de Somsois, dans la Marne, et de Châtillon-sur-Thouet, dans les Deux-Sèvres, toutes deux concernées dans les faits par des sites de stockage temporaire de farines animales, sont également identifiées comme localisation stratégique par le modèle. 


\begin{tabular}{|l|l|l|}
\hline \multicolumn{1}{|c|}{ Commune } & \multicolumn{1}{c|}{ Département } & \multicolumn{1}{c|}{$\begin{array}{c}\text { Capacité de stockage } \\
\text { théorique }\end{array}$} \\
\hline Plounerin & Côtes-d'Armor & 10512 \\
\hline Masseube & Gers & 11576 \\
\hline Pons & Charente-Maritime & 12820 \\
\hline Saint-Germain-de-Marencennes
\end{tabular}

Figure 13. Capacité de stockage des sites de stockage " optimaux » identifiés par le p-médian (par ordre croissant des capacités de stockage)

Outre le calcul de la localisation optimale des sites de stockage par rapport aux usines d'équarrissage et aux cimenteries, le p-médian attribue également un lien entre ces différents points. Son intérêt est, en ce sens, double puisqu'il peut aussi être, dans une certaine mesure, un outil utile pour organiser les transferts de farines animales d'un lieu vers un autre, sa logique étant de toujours privilégier la proximité dans les échanges. Les sites théoriques de Mesgrigny, dans l'Aube, de Bretteville-l'Orgueilleuse, dans le Calvados, et d'Arance, dans les Pyrénées-Atlantiques, sont, à ce titre, de parfaits exemples illustratifs ${ }^{17}$. Selon le modèle, le site de Bretteville-l'Orgueilleuse, dans le Calvados, entreposerait des farines provenant de deux usines d'équarrissage normandes, situées dans l'Orne pour la première et dans le Calvados pour la seconde. La distance moyenne séparant ces deux usines au site est de 96 kilomètres. Les farines auraient ensuite pu être incinérées dans deux cimenteries situées respectivement à Ranville, dans le Calvados, et Saint-Vigor-d'Ymonville, dans la Seine-Maritime. La distance moyenne est ici de 65 kilomètres. Avec cette localisation, les farines animales produites en Normandie n'auraient jamais quitté cette région. Dans les faits, elles furent stockées, au moins en partie, dans le site de Plénée-Jugon, dans les Côtes-d'Armor. Pour les sites de stockage dans le sud-ouest ou dans l'Est de la France, les distances de transit sont supérieures, du

\footnotetext{
${ }^{16}$ A noter que la commune de Plestan fut concernée dans les faits par un site de stockage des farines animales « à haut risque ».

${ }^{17}$ Pour visualiser l'ensemble des liens établis par le modèle, consulter l'annexe $n^{\circ} 35$ de Geffroy A., 2018. La territorialisation de l'action publique en situation de crise. Le cas des farines animales dans le contexte de l'Encéphalopathie Spongiforme Bovine (ESB) en France. Description en bibliographie.
} 
fait de l'implantation moins nombreuse dans ces territoires d'usines d'équarrissage et de cimenteries. Le critère de proximité géographique n'aurait, de ce fait, pas toujours été atteignable du fait des disparités de localisation des ressources spatiales. Malgré cela, les distances de transit calculées par le p-médian pour le site d'Arance et de Mesgrigny restent bien inférieures à celles qui ont pu avoir cours dans la réalité.

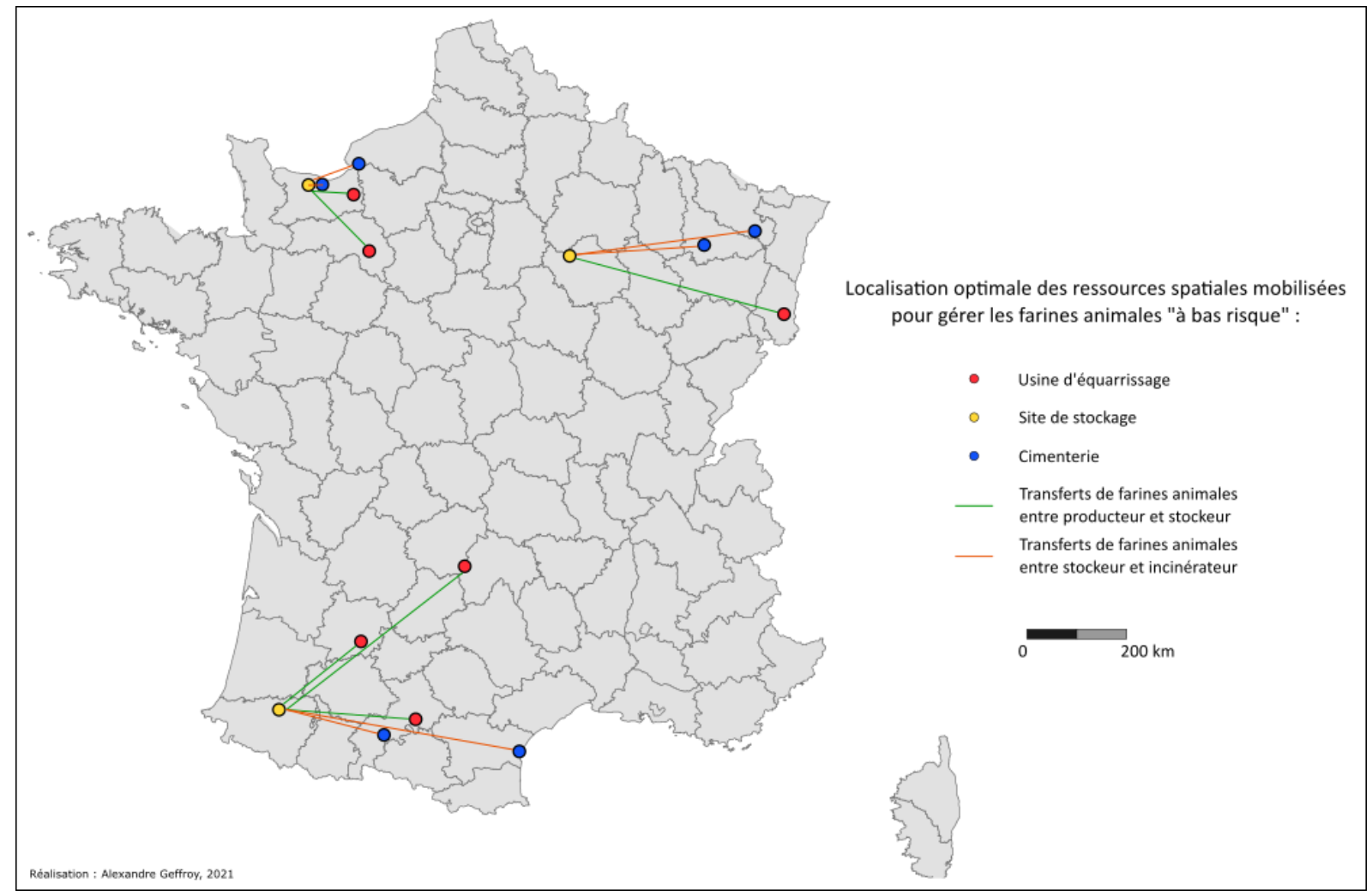

Figure 14. Illustration de transferts théoriques de farines animales entre usines d'équarrissage, sites de stockage et cimenteries déterminés par le p-médian

2.3. Une gestion insuffisamment perçue dans sa dimension nationale et une nonanticipation des volumes à stocker comme facteurs explicatifs des écarts entre stratégies nationales et pratiques locales

De nombreuses raisons peuvent expliquer les écarts observés entre la localisation optimale et la localisation réelle. 


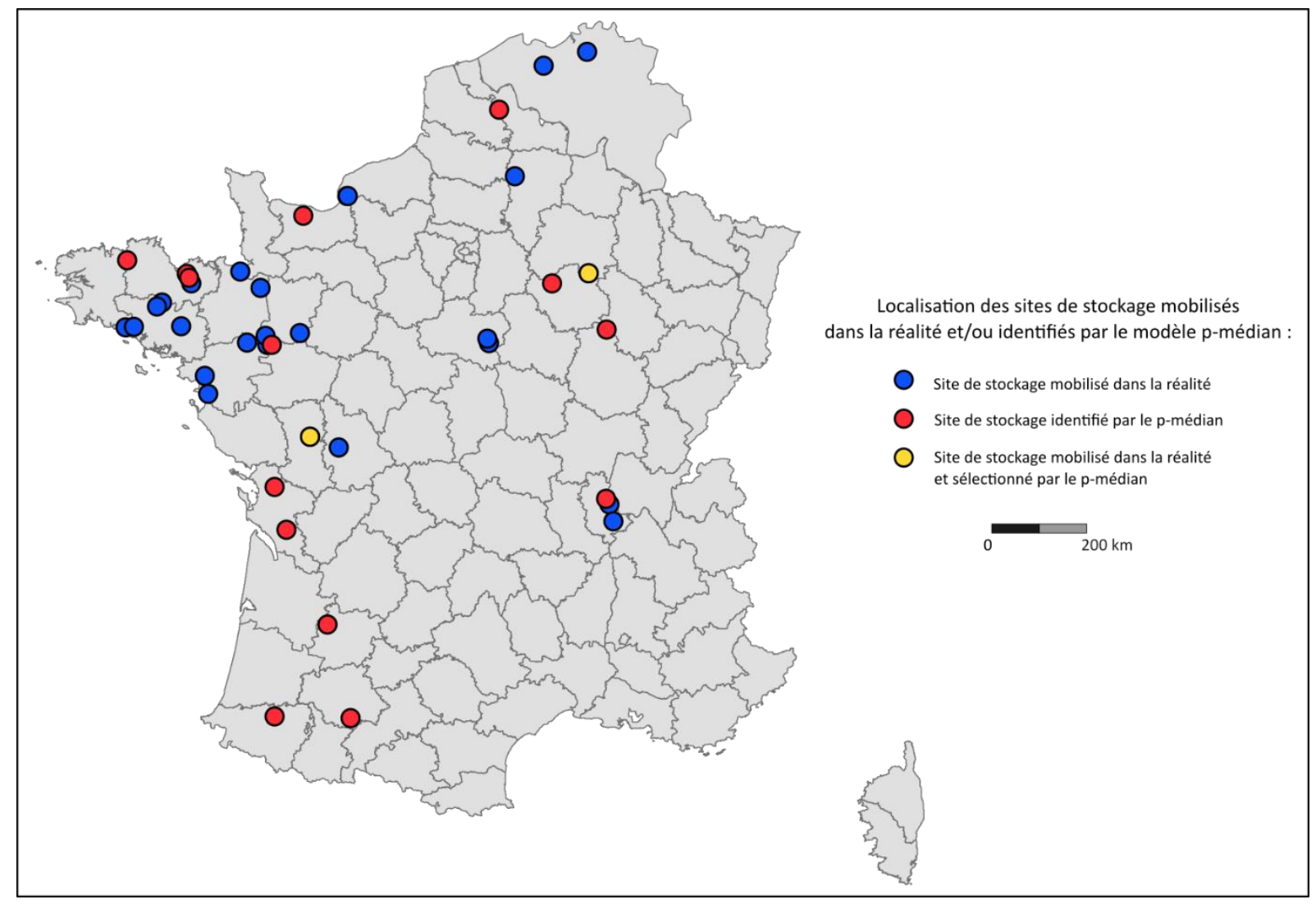

Figure 15. Ecarts dans la localisation des sites de stockage entre la réalité et les résultats du modèle p-médian

La première raison qui peut être invoquée renvoie à la disponibilité des entreprises ICPE lors des périodes de réquisition et des appels d'offres. Comme le relate un ex-membre de la MIEFA, « dans certains cas, ce furent des entreprises avec peu de capacités de stockage qui se mobilisaient mais qui répondaient au critère de proximité tandis que, dans d'autre cas, c'étaient des entreprises avec de grandes possibilités de stockage qui se présentaient à nous ${ }^{18}$. Les préfets de départements étaient donc totalement dépendants de l'offre privée locale et, dans un contexte d'urgence, durent revoir à la baisse les exigences nationales. La gestion réelle de la crise des farines animales semble ainsi être le fruit d'une adaptation des stratégies et normes en fonction des potentialités offertes par les ressources du territoire. Le territoire paraît être, en ce sens, un actant (Lussault, 2010) de la gestion. Il convient donc de nuancer les résultats obtenus par le p-médian. Certaines entreprises sélectionnées par le modèle n'étaient peut-être pas disponibles à l'époque car déjà concernées par d'autres activités. D'autres ont peut-être décliné cette opportunité par crainte de voir se former des mobilisations locales à leur encontre et ainsi voir leur réputation être entachée. L'entreprise située à Somsois, dans le département de la Marne, a, par exemple, vu se former une contestation locale au lendemain de l'attribution du marché public lui reconnaissant la qualité d'entreposeur temporaire de farines animales. A Jussy, dans le département de l'Aisne, une association nommée Non aux Farines Animales s'est constituée dès le 19 décembre 2001 et avait pour objet de « mobiliser la population [...] contre le dépôt de farines animales sur le site imposé par la préfecture à Jussy ». Si les mouvements de contestation présentés ci-dessus n'ont pas entraîné le repli des industriels, peutêtre que cela fut le cas dans d'autres localités. Les préfets durent prendre en compte cette difficulté, facteur qui ne peut être paramétré dans le modèle.

Toutefois, cela ne doit pas occulter le fait que les écarts constatés semblent révéler une certaine absence de perception nationale de la gestion nous amenant à nous interroger sur l'efficacité de

\footnotetext{
${ }^{18}$ Informations tirées d'un entretien conduit le 31/05/2016 avec un ancien membre de la MIEFA.
} 
déléguer les pleins pouvoirs à des niveaux d'action locaux en période de crise affectant tout le territoire métropolitain. En effet, les préfets de département n'ont pas pour vocation de se préoccuper de phénomènes dépassant les frontières de leur circonscription. Cette problématique aurait dû être assurée par les préfets de zone de défense et de sécurité, mobilisés dans le cadre de cette crise par la MIEFA. Il semble néanmoins que ces derniers n'aient pas entièrement joué ce rôle puisqu'ils déléguèrent le soin d'identifier puis de sélectionner les centres de stockage aux préfets des départements de leur circonscription comme le relate une lettre du préfet de la zone de défense ouest, datée du 21 mars 2001, adressée à la MIEFA : «Avant de relancer cette procédure [appel d'offres] dont les délais, tout compris, sont d'environ trois mois, il conviendra de faire le point avec les préfets de département sur les résultats des appels d'offres que certains d'entre eux ont par ailleurs lancés ». L'attribution du pouvoir de sélection à des acteurs locaux apparait être, dès lors, une des sources des décalages observés puisque ce sont principalement les préfets concernés par la production de farines animales dans leur circonscription qui sélectionnèrent des sites de stockage. Il ne semble donc y avoir eu qu'une solidarité nationale toute relative, laissant les préfets concernés dans une situation d'autant plus urgente que les volumes s'accroissaient chaque semaine sans que des sites en dehors de leur périmètre administratif ne soient activés.

Cet accroissement extrêmement rapide des volumes à stocker renvoie à un autre facteur explicatif des décalages observés puisqu'il semblerait que les autorités aient mal anticipé les volumes de farines animales qu'il allait falloir stocker ${ }^{19}$. Comme présenté précédemment, les volumes hebdomadaires de farines «à bas risque » incinérés mirent du temps à atteindre les volumes hebdomadaires produits. En 2001, seules 1000 tonnes, en moyenne, de farines «à bas risque » étaient, par exemple, éliminées chaque semaine. Cette non-anticipation conduisit à une rapide saturation des premiers sites sélectionnés. Les treize premiers centres mobilisés entre novembre et décembre 2000 offraient une capacité d'accueil totale de 241265 tonnes, ceux-ci hébergeant déjà 84020 tonnes au premier janvier 2001. La MIEFA s'aperçut donc que les capacités de stockage allaient être rapidement dépassées, obligeant les autorités à agir dans l'urgence pour identifier de nouveaux établissements à même de recevoir des farines. L'hypothèse de cette non-anticipation est d'ailleurs corroborée par la lettre précédemment citée du préfet de la zone de défense ouest : «La capacité [du site de stockage] de Rogerville [fraîchement sélectionné] représente environ quatre mois de production de farines animales de la zone Ouest. Je vous propose en conséquence de relancer dès à présent une nouvelle procédure d'appel d'offres, sans recourir cette fois à l'urgence ». C'est ainsi que la campagne de sélection des sites de stockage s'étala sur près de deux ans et demi, soit jusqu'en 2003, année où les deux sites Belges furent activés. Cette non-anticipation modifia, de la sorte, le souhait de ne sélectionner qu'un nombre limité d'entreprises.

\section{Conclusion}

L'analyse proposée tend à souligner que la localisation de la gestion de la crise des farines animales, telle qu'elle fut dans la réalité, n'optimisa pas pleinement l'activation des ressources à sa disposition (établissements ICPE, niveaux d'actions). Bien qu'une stratégie nationale fût publiée, son respect fut souvent mis à mal par des enjeux locaux différents exacerbés par l'absence apparente de solidarité entre départements et/ou zones de défense et de sécurité peu ou prou concernés par la production de farines animales dans leur périmètre géographique. Il s'avère donc que la stratégie première de l'Etat central, à savoir confier les pleins pouvoirs de sélection à ses services déconcentrés, ne soit pas la plus adéquate dans le traitement d'une crise de dimension nationale. En

\footnotetext{
${ }^{19}$ Informations tirées d'un entretien conduit le 31/05/2016 avec un ancien membre de la MIEFA : « En France, nous n'avions pas assez de capacités de stockage au vu des volumes produits et de nos capacités d'élimination. Nous étions donc en déficit. II a fallu trouver de nouvelles capacités de stockage. C'est en ce sens que nous nous sommes rapprochés de partenaires belges qui avaient manifesté leur intérêt pour les farines ».
} 
effet, celle-ci tend à introduire dans les processus de gestion une tension entre intérêts locaux et intérêt général. Dans le cadre de la crise des farines animales, cela est rendu visible par le fait que la localisation des sites de stockages ne semble répondre qu'à une logique de proximité aux producteurs de farines animales, délaissant la logique réticulaire et une vision à long terme par l'anticipation des flux à destination des cimentiers. Ce ne sont ainsi presque que des préfets de départements concernés par la production de farines animales dans leur circonscription qui participèrent au processus de sélection des centres d'entreposage. La forte concentration de ces centres dans le Grand ouest français ne permit pas de ménager les capacités d'élimination des incinérateurs locaux. Au contraire, leurs capacités furent très vite saturées, obligeant les autorités nationales à accepter des transferts sur de très longues distances, pourtant contraires à leur stratégie initiale. Il semblerait, de fait, plus judicieux lors d'une crise nationale d'imposer un acteur à même d'orienter stratégiquement la localisation des infrastructures nécessaires à la résorption d'une crise. Les modèles de localisation-affectation peuvent, en ce sens, être de très bons outils à utiliser lors des phases de préparation et d'anticipation de la gestion d'une crise. Ils permettent, en effet, de pouvoir cibler, en fonction des critères et enjeux de la situation à traiter, les lieux où il serait le plus judicieux d'implanter ces dites infrastructures. Dans ce cadre, le pouvoir de sélection pourrait revenir aux services déconcentrés de l'Etat qui ont une connaissance plus fine des terrains ciblés par le modèle. Par cette anticipation et cette prise en compte de la dimension nationale d'une crise, il serait possible de dépasser les absences de solidarité interterritoriale qui caractérisent la gestion des farines animales mais qui sont également bien documentées face à d'autres phénomènes critiques comme la gestion des inondations par exemple (Douvinet et al, 2010 ; Lorant-Plantier et Pech, 2011).

\section{Bibliographie}

BARAY Jérôme, Localisation commerciale multiple : une application du traitement du signal et du modèle p-médian au développement d'un réseau de magasins de produits biologiques, Thèse, spécialité Sciences de gestion, 2002, Université de Rennes 1, 379 p.

BARBIER Marc, «Une interprétation de la constitution de l'ESB comme problème public européen », in Revue internationale de politique comparée, no 2, vol. 10, 2003, p. 233

BASSETT Thomas J. et GAUTIER Denis, «Territorialisation et pouvoir: la Political Ecology des territoires de conservation et de développement : Introduction », in EchoGéo, no 29, 18 novembre 2014, p. 1-9.

BECK Corinne, «Sociétés et ressources : les crises en question » in Temps et espaces des crises de l'environnement, BECK Corinne, LUNGINBÜHL Yves et MUXART Tatiana (dir.), Paris, Editions QUAE GIE, 2006, p. 9-14

BEGUIN Hubert, «La localisation des activités banales » in Encyclopédie de géographie, BAILLY Antoine, FERRAS Robert et PUMAIN Denise (dir.), 2. éd., Paris, Economica, 1995, p. 497-513.

BEGUIN Hubert, HANSEN Pierre et THISSE Jacques-François, « Où construire les équipements collectifs ? », in Recherches Economiques de Louvain, no 48, 1982, p. 211-217.

BIZET, Jean, Rapport de la commission d'enquête sur les conditions d'utilisation des farines animales dans l'alimentation des animaux d'élevage et les conséquences qui en résultent pour la santé des consommateurs, Paris, Sénat, 2001.

CORTES Geneviève et PESCHE Denis, «Territoire multisitué », in L’Espace géographique, no 4, vol. 42, 2013, p. 289-292.

DASKIN Mark S., Network and discrete location: models, algorithms, and applications, New York, Wiley, « WileyInterscience series in discrete mathematics and optimization », 1995, $498 \mathrm{p}$.

DAUTUN Carole, TIXIER Jérôme, CHAPELAIN Jean, FONTAINE François et DUSSERRE Gilles, « Le traitement de l'incertitude en gestion de crise : mise en place d'une veille stratégique du territoire », Colloque de maitrise des risques et sûreté de fonctionnement « Risques et performances », Lille, 2006.

DAVIS A. J., JENNY A. L., MILLER L. D. (1991), « Diagnostic Characteristics of Bovine Spongiform Encephalopathy », Journal of Veterinary Diagnostic Investigation, p. 266-271. DOI : $10.1177 / 104063879100300318$ 
D'ERCOLE Robert, HARDY Sébastien, METZGER Pascale, ROBERT Jérémy et GLUSKI Pauline, « Les dimensions spatiales et territoriales de la gestion de crise à Lima », in VertigO, Volume 12 Numéro 1, 25 juin 2012.

DOUVINET Johnny, DEFOSSEZ Stéphanie, ANSELLE Arnaud et DENOLLE Anne-Sophie, «Les maires face aux plans de prévention du risque inondation (Ppri), in L'Espace géographique, no 1, vol. 40, 2010, p. 31-46

DUBOIS-MAURY Jocelyne et CHALINE Claude, Les risques urbains, Paris, A. Colin, 2004, 208 p

GEFFROY Alexandre, La territorialisation de l'action publique en situation de crise. Le cas des farines animales dans le contexte de l'Encéphalopathie Spongiforme Bovine (ESB) en France, Thèse, spécialité Géographie, 2018, Université Rouen Normandie, 486 p.

GEFFROY Alexandre et ELIOT Emmanuel, « La gestion de la crise des farines animales en France (2000-2009) dans le contexte de l'Encéphalopathie Spongiforme Bovine (ESB) : temporalités, acteurs et niveaux d'action ", L'Espace Politique [En ligne], $36 \mid$ 2018-3, mis en ligne le 27 mai 2019, http://journals.openedition.org/espacepolitique/5540 ; DOI : https://doi.org/10.4000/espacepolitique.5540

GILBERT Claude (éd.), Risques collectifs et situations de crise : apports de la recherche en sciences humaines et sociales, Paris, L'Harmattan, 2002, 340 p.

GONIN Alexis, «Les éleveurs face à la territorialisation des brousses : repenser le foncier pastoral en Afrique de l'Ouest », in Annales de géographie, no 1, vol. 707, 2016, p. 28-50.

HAKIMI Seifollah L., «Optimum Locations of Switching Centres and the Absolute Centres and Medians on a Graph », in Operations Research, no 3, vol. 12, 1964, p. 450-459

HORBY Peter, «Rendering Beef Safe », in Clinical Infectious Disease, no 34, 2002, p. 129.

LACQUEMENT Guillaume et CHEVALIER Pascal, «Capital territorial et développement des territoires locaux, enjeux théoriques et méthodologiques de la transposition d'un concept de l'économie territoriale à l'analyse géographique », in Annales de géographie, no 5, vol. 711, 2016, p. 490.

LAJARGE Romain, PECQUEUR Bernard, LANDEL Pierre-Antoine et LARDON Sylvie, Ressources territoriales : gouvernance et politiques publiques, RessTerr Rhône-Alpes \& RessTerr Auvergne, 2012.

LE GALES Patrick, " Gouvernance » in Dictionnaire de la géographie et de l'espace des sociétés, LEVY, JACQUES et LUSSAULT, MICHEL (dir.), Paris, Belin, 2013, p. 457-461.

LÖDEL Roland, Risques liés au stockage des farines animales, Rapport final, Ministère de l'Environnement, INERIS, 1997.

LORANT-PLANTIER Emilie et PECH Pierre, «La gestion du risque inondation en France vecteur de territorialité : l'exemple de l'entente Oise-Aisne, bassin versant de 1'Oise », in Annales de géographie, no 2, vol. 678, 2011, p. 193-203.

LORD PHILLIPS OF WORTH MATRAVERS, BRIDGEMAN June et FERGUSON-SMITH Malcolm, The BSE Inquiry: The Report. The Inquiry into BSE and variant CJD in the United Kingdom, Londres, 2000.

LUSSAULT Michel, « Ce que la géographie fait au(x) monde(s) », in Tracés, no 10, 30 novembre 2010, p. 241-251.

METZGER Pascal, ROBERT Jérémy, SIERRA Alexis, D’ERCOLE Robert, HARDY Sébastien et GLUSKI Pauline, «Dimensions spatiales et territoriales de la gestion de crise : les ressources de décision et d'intervention à Lima et Callao », in Revue Géographique de l'Est, Volume 53 Numéro 1-2, 2013

NOVAKOFSKI J., BREWER M. S., MATEUS-PINILLA N., KILLEFER J. et MCCUSKER R. H., « Prion biology relevant to bovine spongiform encephalopathy », in Journal of Animal Science, no 6, vol. 83, 1 juin 2005, p. 14551476.

ROBERT Jérémy, Pour une géographie de la gestion de crise : de l'accessibilité aux soins d'urgence à la vulnérabilité du territoire de Lima, Thèse, spécialité Géographie, 2012, Université Grenoble, 549 p.

PEETERS Dominique et THOMAS Isabelle, «Distance-Lp et localisations optimales. Simulations sur un semis aléatoire de points », in Les cahiers Scientifiques du Transport, no 31, 1997, p. 55-70.

PELUSO Nancy Lee, « Seeing property in land use: Local territorializations in West Kalimantan, Indonesia », in Geografisk Tidsskrift-Danish Journal of Geography, n o 1, vol. 105, janvier 2005, p. 1-15.

QUERRIAU Xavier, KISSIYAR Mohamed, PEETERS Dominique et THOMAS Isabelle, « Localisation optimale d'unités de soins dans un pays en voie de développement : analyse de sensibilité », in Cybergeo, 24 août 2004. 
RONDINELLI Dennis A., NELLIS John R. et CHEEMA Shabbir G., Decentralization in Developping Countries. A Review of Recent Experience, s.l., World Bank Staff Working Papers, 1983.

DASKIN Mark S., Network and discrete location: models, algorithms, and applications, New York, Wiley, « WileyInterscience series in discrete mathematics and optimization », 1995, $498 \mathrm{p}$.

SACK, R. (1986) Human Territoriality: Its Theory and History. Vol. 14, Cambridge University Press, Cambridge, 1618.

SOULIES Dorian, Le secours à personne : spatialiser, modéliser, outil d'aide à la décision : méthode d'optimisation de la localisation des moyens de secours à personne dans le cadre de la réalisation de documents de planification: application au département des Alpes-Maritimes, Thèse, spécialité Géographie, Université Nice Sophia Antipolis, $2015,302 \mathrm{p}$

SUZUKI Atsuo et DREZNER Zvi, "The p-center location problem in area" in Location Science, Issues 1-2, vol 4, may-august 1996, p. 69-82

WOLFER Bernard, «Une décision sous influence: l'interdiction des farines », in Dossier de l'Environnement de l'INRA, no 28, 2004, p. 136-148. 